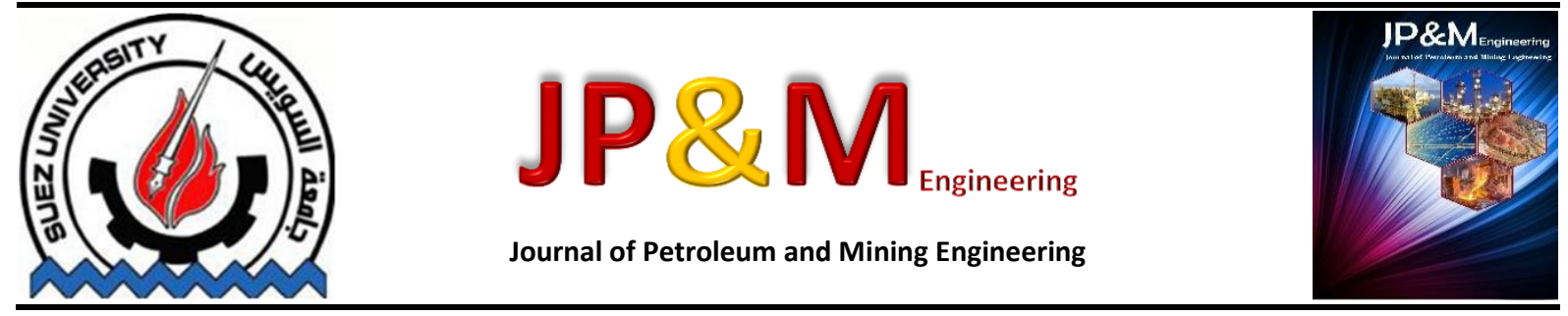

\title{
A Study on Gas Compressibility Factor for Gas-Condensate Systems
}

\author{
Ali M. Wahba *, Hamid M. Khattab, and Ahmed A. Gawish \\ Petroleum Engineering Department, Faculty of Petroleum and Mining Engineering, Suez University- Egypt. \\ *Corresponding author ali.mustafa1992@yahoo.com
}

\begin{abstract}
Gas compressibility factor is the most important gas property. Its value is required in many petroleum engineering calculations. There are many different sources of gas compressibility factor value such as experimental measurements, equations of state, charts, tables, intelligent approaches and empirical correlations methods. In absence of experimental measurements of gas compressibility factor values, it is necessary for the petroleum engineer to find an accurate, quick and reliable method for predicting these values. This study presents a new gas compressibility factor explicit empirical correlation for gas-condensate reservoir systems above dew point pressure. This new correlation is more robust, reliable and efficient than the previously published explicit empirical correlations. It is also in a simple mathematical form. The predicted value using this new correlation can be used as an initial value of implicit correlations to avoid huge number of iterations. This study also presents evaluation of the new and previously published explicit correlations.
\end{abstract}

Natural Gas,; Compressibility Factor; Empirical Correlation Validation. important property of natural gas. It accounts for how much the real gas behavior deviates from the ideal gas behavior at given condition. According to real gas law, it is expressed as a function of pressure, volume, and temperature as follows:

$Z=\frac{P V}{n R T}$

Since 1942, Standing and Katz [1] gas Z-factor chart has become a standard in petroleum industry which is used to estimate gas compressibility factor. It is based on the principle of corresponding states. This principle states that two substances at the same conditions referenced to critical pressure and critical temperature will have similar properties. According to the principle of corresponding states, gas compressibility factor is expressed as a function of reduced pressure and reduced temperature as follows:

$$
\begin{gathered}
Z=f\left(P_{r}, T_{r}\right) \\
\text { Where: } \quad P_{r}=\frac{P}{P_{c}} \\
T_{r}=\frac{T}{T_{c}}
\end{gathered}
$$

For gas mixture, critical and reduced properties are replaced with pseudo-critical and pseudo-reduced properties. The accuracy of pseudo-critical properties calculation will affect the accuracy of gas Z-factor estimation.

Several methods for calculation of natural gas pseudo-critical pressure and temperature for gascondensate reservoir systems have been developed.
Gas compressibility factor which is also called gas deviation factor or simply gas Z-factor is the most 
These methods are divided into two categories. In this study, Sutton (1985) [2] modification to StewartBurkhardt-Voo [3] mixing rules (SSBV mixing rules), Corredor et al. (1992) [4] mixing rules, Piper et al. (1993) [5] mixing rules, Al-Sharkawy et al. (2000) [6] mixing rules and Al-Sharkawy (2004) [7] mixing parameters are used when natural gas composition is known while Sutton (1985) [2] empirical correlations, Piper et al. (1993) [5] mixing rules, El-Sharkawy-ElKamel (2000) [8] empirical correlations and Sutton (2005) [9] empirical correlations used when natural gas composition is unknown. For some of these methods, the critical properties of all components of the gas are required. The critical properties of pure components are well known as given in Table 1. Gascondensate gases contain hydrocarbon-plus fractions such as heptanes-plus $\left(C_{7^{+}}\right)$fraction. So, the critical properties of the hydrocarbon-plus fractions must be estimated. Several correlations have been developed to estimate the critical properties of the hydrocarbonplus fractions. Kesler and Lee (1976) [10] correlations, Whitson (1987) [11] correlation and Riazi and Daubert (1987) [12] correlations are used in this study. Gascondensate gases also contain impurities such as hydrogen sulfide $\left(\mathrm{H}_{2} \mathrm{~S}\right)$, carbon dioxide $\left(\mathrm{CO}_{2}\right)$, nitrogen $\left(\mathrm{N}_{2}\right)$ and water vapor $\left(\mathrm{H}_{2} \mathrm{O}\right)$ which affect the pseudo-critical pressure and temperature values. Several correlations have been developed to account for the presence of these impurities. Wichert and Aziz (1972) [13] correction method, Modified Wichert and Aziz [9] correction method, Standing (1981) [14] correction method and Casey [15] correction method are used in this study. The corrected pseudo-critical pressure and temperature are used to calculate the pseudo-reduced pressure and temperature which are the parameters of gas compressibility factor empirical correlations. All of the above mentioned methods are summarized in Appendix A.

Gas compressibility factor empirical correlations are divided into two categories: implicit and explicit empirical correlations. Implicit empirical correlations require iterative solution methods. The most used implicit empirical correlations are Hankinson Thomas and Phillips (1969) [16], Hall-Yarborough (1973) [17], Dranchuk-Purvis-Robinson (1974) [18], DranchukAbu-Kassem (1975) [19] and Hall and Iglesias-Silva (2007) [20] correlations. But, explicit empirical correlations do not require iterative solution methods. They are used directly to calculate gas compressibility factor. The most used previously published explicit empirical correlations are Papay (1968) [21], Beggs and Brill (1973) [22], Gopal (1977) [23], Burnett (1979) [24], Kumar (2004) [25], Bahadori et al. (2007) [26], Al-Anazi and Al-Quraishi (2010) [27], Azizi et al. (2010) [28], Heidaryan-SalarabadiMoghadasi (2010) [29], Heidaryan-Moghadasi-Rahimi (2010) [30], Shokir et al. (2012) [31], Sanjari and Nemati Lay (2012) [32], M.A. Mahmoud (2013) [33] and Niger Delta (2013) [34] correlations. These explicit empirical correlations are summarized in Appendix B.

\section{Data Acquisition}

Huge data points were collected to achieve the main objectives of this study. They were divided into two sets according to the source of data points: general data set and specific data set. General data set consists of five thousand, nine hundred and forty data points of gas Z-factor values as a function of pseudoreduced pressure and temperature. They were the result of Standing and Katz chart digitization done by Poettmann and Carpenter. Statistical distributions such as maximum, minimum, mean, median and range of this data set are shown in Table 2. Specific data set consists of seven hundred and twenty one data points of gas Z-factor values as a function of pseudo-reduced pressure and temperature. These data points are measured at pressures above the dew point pressures of the gas-condensate reservoir systems. They were prepared from data collected from unpublished gas-condensate gas PVT reports. This collected data was reservoir pressure and temperature, mole fraction of gas chemical composition and gas specific gravity. The statistical distributions such as maximum, minimum, mean, median and range of this collected data are shown in Table 3.

\section{Research Methodology}

To achieve the objectives of this study, MATLAB Surface Fitting Tool (sftool) was used to develop a new explicit empirical correlation of gas compressibility factor. EXCEL sheets were used to validate the performance of this new correlation. EXCEL sheets were also used to evaluate and grade the performance of this new and other explicit correlations. These validation and evaluation were performed using statistical error analysis such as average absolute percent relative error (AARE\%), residual sum of squares (RSS), root mean square error (RMSE), standard deviation (SD) and coefficient of determination (R2) and also with using graphical analysis such as cross plot parity line.

\section{Results and Discussion}

To develop the new explicit empirical correlation, 4000 data points from general data set are entered in MATLAB Surface Fitting Tool (sftool). Fig. 1 shows the surface plot of the new correlation which has the following form:

$$
Z=-0.1284+0.3098 T_{p r}+0.1427 P_{p r}+
$$
$0.3222 T_{p r}^{2}-0.1571 T_{p r} P_{p r}+0.009456 P_{p r}^{2}-$ $0.0963 T_{p r}^{3}+0.02993 T_{p r}^{2} P_{p r}-$ $0.00002458 T_{p r} P_{p r}^{2}-0.0002861 P_{p r}^{3}$

This new empirical correlation is in a simple mathematical form. In which, the gas compressibility factor is a function of pseudo-reduced pressure and temperature. Figure 2 shows the training of this new empirical correlation using 4000 data points from general data set. The statistical parameters values of this training are: $\mathrm{RSS}=0.6429, \mathrm{RMSE}=0.0127, \mathrm{AARE} \%$ $=0.904, \mathrm{SD}=1.1631$ and $\mathrm{R} 2=0.9964$. 


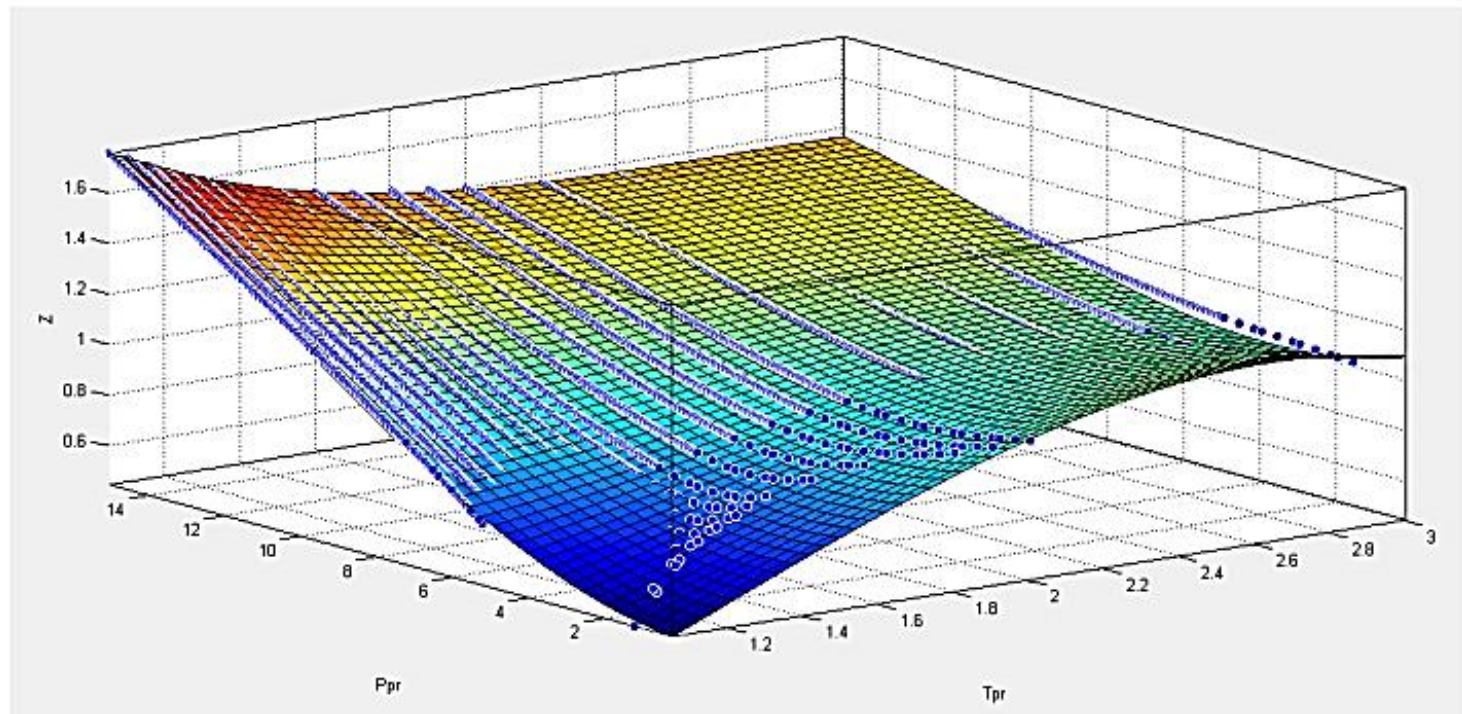

Figure 1 Surface plot of the new proposed Z-factor explicit empirical correlation.

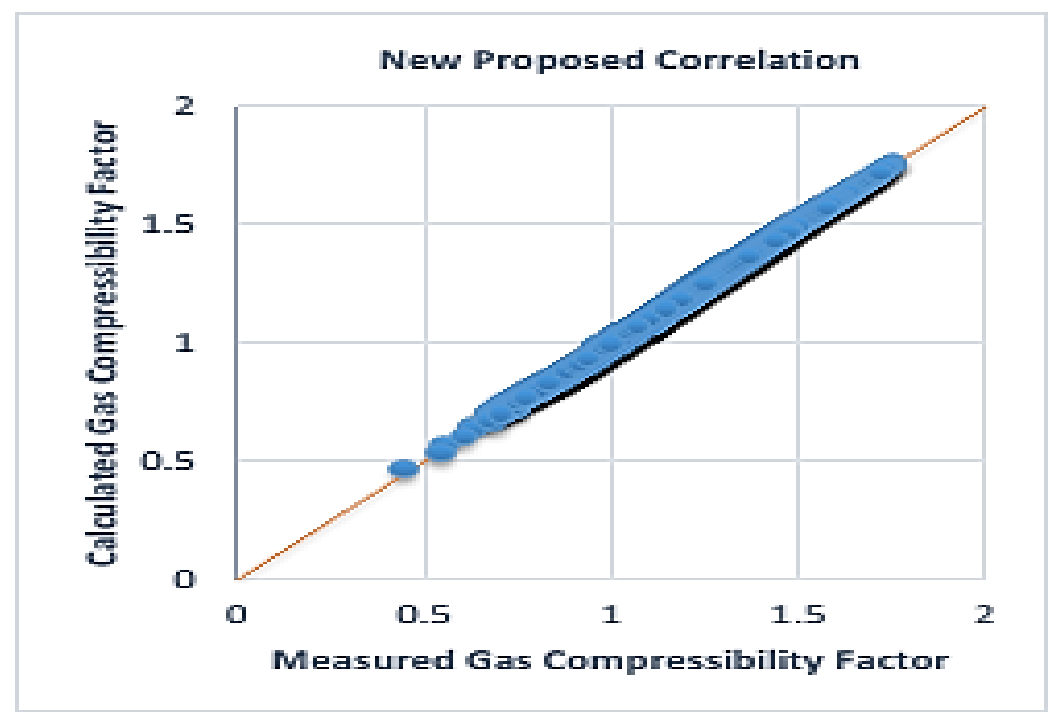

Figure 2 Accuracy of the new proposed Z-factor explicit empirical correlation for training.

\section{Evaluation and Validation}

The new and other explicit empirical correlations can be used to predict the Z-factor of gas-condensate gases depending on the choice of the correct gas pseudo-critical pressure and temperature calculation method. There are several methods to calculate gas pseudo-critical pressure and temperature and accessories methods to account for the presence of heptanes-plus fraction and impurities in gascondensate gases as mentioned above in literature review section. From these methods, twelve methods are formed when gas composition is known and six methods are formed when gas composition is unknown. These methods are evaluated using 721 data points of specific data set. The statistical parameters values of this evaluation are shown in Appendix C from Table C-1 to Table C-18. As shown from these tables, six explicit empirical correlations have high coefficient of determination (R2) values. The cross plots of these correlations when using Sutton (1985), Standing, Wichert \& Aziz and Casey method for Ppc and Tpc calculations are shown in Appendix C from Figure C-1 to Figure C-6.

\section{Conclusions}

The conclusions emanating from this study are as follows:

- A new explicit empirical correlation for Z-factor is obtained in simple mathematical form.

- The obtained correlation provides better predictions of gas Z-factor values than other explicit empirical correlations. As it gives the highest accuracy when using any method for calculating gas pseudo-critical pressure and temperature either when gas composition is known or unknown except for using El-Sharkawy empirical correlations for calculating gas pseudo-critical pressure and temperature when gas composition is unknown because of the accuracy of these correlations.

- The proposed correlation is recommended for the following pseudo-reduced pressure and temperature ranges for gas-condensate reservoir systems above dew point pressure: 
$1<P_{p r} \leq 15$

$1.05 \leq T_{p r} \leq 3.0$
- The predicted gas Z-factor value using the new correlation can be used, out of this new correlation recommended ranges, as an initial value of implicit correlations to avoid huge number of iterations.

Table 1 Physical properties of defined components.

\begin{tabular}{|c|c|c|c|} 
Component & Molecular weight & Critical pressure, psia & Critical temperature, ${ }^{\circ} \boldsymbol{R}$ \\
\hline $\boldsymbol{H}_{2} \boldsymbol{s}$ & 34.08 & 1306 & 1306 \\
\hline $\boldsymbol{C o}_{\mathbf{2}}$ & 44.01 & 1071 & 1071 \\
\hline $\boldsymbol{N}_{\mathbf{2}}$ & 28.0134 & 493 & 493 \\
\hline $\boldsymbol{C}_{\mathbf{1}}$ & 16.043 & 667.8 & 667.8 \\
\hline $\boldsymbol{C}_{\mathbf{2}}$ & 30.07 & 707.8 & 707.8 \\
\hline $\boldsymbol{C}_{\mathbf{3}}$ & 44.097 & 616.3 & 616.3 \\
\hline $\mathbf{i}-\boldsymbol{C}_{\mathbf{4}}$ & 58.123 & 529.1 & 529.1 \\
\hline $\mathbf{n}-\boldsymbol{C}_{\mathbf{4}}$ & 58.123 & 550.7 & 550.7 \\
\hline $\mathbf{i}-\boldsymbol{C}_{\mathbf{5}}$ & 72.15 & 490.4 & 490.4 \\
\hline $\mathbf{n}-\boldsymbol{C}_{\mathbf{5}}$ & 72.15 & 488.6 & 488.6 \\
\hline $\boldsymbol{C}_{\mathbf{6}}$ & 86.177 & 436.9 & 436.9 \\
\hline
\end{tabular}

Table 2 Satistical distributions of general data set.

\begin{tabular}{|c|c|c|c|c|c|}
\hline & Minimum & Maximum & Mean & Median & Range \\
\hline $\boldsymbol{T}_{\boldsymbol{p r}}$ & 1.05 & 3 & 1.7375 & 1.55 & 1.95 \\
\hline $\boldsymbol{P}_{\boldsymbol{p r}}$ & 0.2 & 15 & 7.6 & 7.6 & 14.8 \\
\hline $\boldsymbol{Z}-\boldsymbol{f a c t o r}$ & 0.251 & 1.753 & 1.051966 & 1.0345 & 1.502 \\
\hline
\end{tabular}

Table 3 Statistical distributions of specific data set.

\begin{tabular}{|c|c|c|c|c|c|} 
& Minimum & Maximum & Mean & Median & Range \\
\hline$T_{\text {res }}$ o & 147 & 309 & 269.27 & 281 & 162 \\
\hline$P_{\text {res }} p$ psig & 2728 & 9247 & 7037.85 & 7593 & 6519 \\
\hline$H_{2^{5}}$ & 0 & 5.98 & 1.65 & 0.72 & 5.98 \\
\hline$C_{0_{2}}$ & 0.12 & 3.93 & 2.42 & 2.36 & 3.81 \\
\hline$N_{2}$ & 0.17 & 18.29 & 8.42 & 9.32 & 18.12 \\
\hline$C_{1}$ & 65.07 & 91.7 & 72.00 & 69.26 & 26.63 \\
\hline$C_{2}$ & 3.37 & 10.5 & 6.63 & 5.91 & 7.13 \\
\hline$C_{3}$ & 1.3 & 4.97 & 2.80 & 2.47 & 3.67 \\
\hline$i \cdot C_{4}$ & 0.3 & 0.95 & 0.55 & 0.51 & 0.65 \\
\hline$n \cdot C_{4}$ & 0.31 & 1.87 & 1.04 & 0.95 & 1.56 \\
\hline$i \cdot C_{5}$ & 0.16 & 0.77 & 0.40 & 0.40 & 0.61 \\
\hline$n \cdot C_{5}$ & 0.09 & 0.78 & 0.41 & 0.36 & 0.69 \\
\hline$C_{6}$ & 0.15 & 1.25 & 0.55 & 0.51 & 1.10 \\
\hline$C_{7+}$ & 0.53 & 7.65 & 3.14 & 2.71 & 7.12 \\
\hline$M_{C_{7+}}$ & 122 & 171 & 146.46 & 144 & 49 \\
\hline$\gamma_{C_{7+}}$ & 0.73 & 0.81 & 0.79 & 0.79 & 0.08 \\
\hline$\gamma_{g}$ & 0.67 & 1.19 & 0.88 & 0.86 & 0.52 \\
\hline
\end{tabular}


Nomenclature

\begin{tabular}{|c|c|c|}
\hline AARE $\%$ & $=$ & $\begin{array}{l}\text { Average absolute percent relative error, } \\
\quad \%\end{array}$ \\
\hline $\mathrm{E}_{\mathrm{J}}$ & $=$ & Sutton SBV parameter, ${ }^{\circ} \mathrm{R} / \mathrm{psia}$ \\
\hline $\mathrm{E}_{\mathrm{K}}$ & $=$ & Sutton SBV parameter, ${ }^{\circ} \mathrm{R} / \mathrm{psia}^{0.5}$ \\
\hline $\mathrm{J}$ & $=$ & $\mathrm{SBV}$ parameter, ${ }^{\circ} \mathrm{R} / \mathrm{psia}$ \\
\hline $\mathrm{K}$ & $=$ & $\mathrm{SBV}$ parameter, ${ }^{\circ} \mathrm{R} / \mathrm{psia}^{0.5}$ \\
\hline M & $=$ & Molecular weight, lb/lb-mole \\
\hline $\mathrm{M}_{\mathrm{air}}$ & $=$ & $\begin{array}{l}\text { Apparent molecular weight of the air } \\
\text { which has the value } 28.964 \mathrm{lb} / \mathrm{lb}- \\
\text { mole }\end{array}$ \\
\hline $\mathrm{M}_{\mathrm{Co}_{2}}$ & $=$ & Molecular weight of Carbon Dioxide \\
\hline $\mathrm{M}_{\mathrm{C}_{7+}}$ & $=$ & $\begin{array}{l}\text { Molecular weight of heptanes-plus } \\
\text { component }\end{array}$ \\
\hline $\mathrm{M}_{\mathrm{H}_{\curvearrowright} \mathrm{S}}$ & $=$ & Molecular weight of Hydrogen Sulfide \\
\hline $\mathrm{M}_{\mathrm{N}_{2}}$ & $=$ & Molecular weight of Nitrogen \\
\hline $\mathrm{N}$ & $=$ & Number of moles of gas, lb-mole \\
\hline $\mathrm{P}$ & $=$ & Absolute pressure, psia \\
\hline $\mathrm{P}_{\mathrm{c}}$ & $=$ & Critical pressure, psia \\
\hline $\mathrm{P}_{\mathrm{cor}_{\mathrm{Co}}}$ & $=$ & Critical pressure of Carbon Dioxide \\
\hline $\mathrm{P}_{\mathrm{c}_{\mathrm{H}} \mathrm{c}}$ & $=$ & Critical pressure of Hydrogen Sulfide \\
\hline $\mathrm{P}_{\mathrm{c}_{\mathrm{N}}}$ & $=$ & Critical pressure of Nitrogen \\
\hline $\mathrm{P}_{\mathrm{pc}}$ & $=$ & Pseudo-critical pressure, psia \\
\hline $\mathrm{P}_{\mathrm{pc}_{\mathrm{HC}}}$ & $=$ & $\begin{array}{l}\text { Pseudo-critical pressure of hydrocarbon } \\
\text { portion }\end{array}$ \\
\hline $\mathrm{P}_{\mathrm{pr}}$ & $=$ & Pseudo-reduced pressure, dimensionless \\
\hline $\mathrm{P}_{\mathrm{r}}$ & $=$ & Reduced pressure, dimensionless \\
\hline PVT & $=$ & Pressure, volume and temperature \\
\hline $\mathrm{R}$ & $=$ & $\begin{array}{l}\text { The universal gas constant which has the } \\
\text { value } 10.73 \text { psia. } \mathrm{ft}^{3} / \mathrm{lb} \text {-mole/ }{ }^{\circ} \mathrm{R}\end{array}$ \\
\hline $\mathrm{R}^{2}$ & $=$ & Coefficient of determination, fraction \\
\hline RMSE & $=$ & Root mean square error, fraction \\
\hline RSS & $=$ & Residual sum of squares, fraction \\
\hline SD & $=$ & Standard deviation, $\%$ \\
\hline $\mathrm{T}$ & $=$ & Absolute temperature, ${ }^{\circ} \mathrm{R}$ \\
\hline $\mathrm{T}_{\mathrm{b}}$ & $=$ & Boiling temperature, ${ }^{\circ} \mathrm{R}$ \\
\hline $\mathrm{T}_{\mathrm{c}}$ & $=$ & Critical temperature, ${ }^{\circ} \mathrm{R}$ \\
\hline $\mathrm{T}_{\mathrm{c}_{\operatorname{co}},}$ & $=$ & Critical temperature of Carbon Dioxide \\
\hline $\mathrm{T}_{\mathrm{c}_{\mathrm{H}} \mathrm{c}}$ & $=$ & Critical temperature of Hydrogen Sulfide \\
\hline $\mathrm{T}_{\mathrm{c}_{\mathrm{N}}}$ & $=$ & Critical temperature of Nitrogen \\
\hline $\mathrm{T}_{\mathrm{pc}}$ & $=$ & Pseudo-critical temperature, ${ }^{\circ} \mathrm{R}$ \\
\hline $\mathrm{T}_{\mathrm{pc}_{\mathrm{HC}}}$ & $=$ & $\begin{array}{l}\text { Pseudo-critical temperature of } \\
\text { hydrocarbon portion }\end{array}$ \\
\hline $\mathrm{T}_{\mathrm{pr}}$ & $=$ & $\begin{array}{l}\text { Pseudo-reduced temperature, } \\
\text { dimensionless }\end{array}$ \\
\hline $\mathrm{T}_{\mathrm{r}}$ & $=$ & Reduced temperature, dimensionless \\
\hline $\mathrm{V}$ & $=$ & Volume, $\mathrm{ft}^{3}$ \\
\hline $\mathrm{W}_{\mathrm{HC}}$ & $=$ & Weight fraction of hydrocarbon portion \\
\hline $\mathrm{W}_{\mathrm{NHC}}$ & $=$ & $\begin{array}{l}\text { Weight fraction of non-hydrocarbon } \\
\text { portion }\end{array}$ \\
\hline $\mathrm{y}_{\mathrm{Co}_{2}}$ & $=$ & Mole fraction of Carbon Dioxide \\
\hline $\mathrm{y}_{\mathrm{C}_{7+}}$ & $=$ & $\begin{array}{l}\text { Mole fraction of heptanes-plus } \\
\text { component }\end{array}$ \\
\hline $\mathrm{y}_{\mathrm{HC}}$ & $=$ & Mole fraction of hydrocarbon portion \\
\hline $\mathrm{y}_{\mathrm{H}_{\mathrm{s}} \mathrm{s}}$ & $=$ & Mole fraction of Hydrogen Sulfide \\
\hline
\end{tabular}

$\mathrm{y}_{\mathrm{i}}=$ Mole fraction of component $\mathrm{i}$ in the gas mixture

$\mathrm{y}_{\mathrm{N}_{2}}=$ Mole fraction of Nitrogen

$\mathrm{Z}=$ Gas deviation factor, dimensionless

Greek symbols

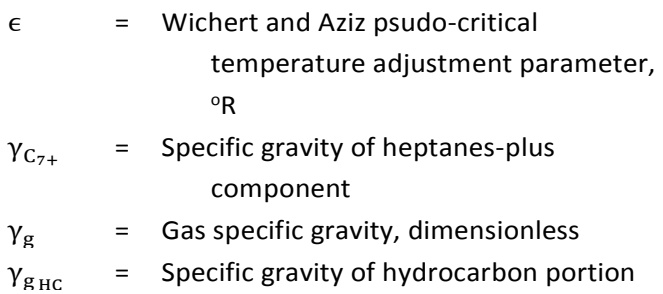

\section{References}

[1] Standing, M.B. and Katz, D.L.: "Density of Natural Gases," Trans. AIME, 146, 1942, pp. 140-149.

[2] Sutton, R.P.: "Compressibility Factors for HighMolecular-Weight Reservoir Gases," paper SPE 14265 presented at the $60^{\text {th }}$ SPE Annual Technical Conference and Exhibition, Las Vegas, NV, September 22-25, 1985, pp. 22-25.

[3] Stewart, W.F., Burkhardt, S.F. and Voo, D.: "Prediction of Pseudo-Critical Parameters for Mixtures," paper presented at the AIChE Meeting, Kansas City, MO., May 18, 1959

[4] Corredor, J.H., Piper, L.D. and McCain, W.D. Jr.: "Compressibility Factors for Naturally Occurring Petroleum Gases," paper SPE 24864 presented at the $67^{\text {th }}$ SPE Annual Technical Conference and Exhibition, Washington, DC, October 4-7, 1992.

[5] Piper, L.D., McCain Jr. and Corredor, J.H.: "Compressibility Factors for Naturally Occurring Petroleum Gases," paper SPE 26668 presented at the $68^{\text {th }}$ SPE Annual Technical Conference and Exhibition, Houston, TX, October 3-6, 1993.

[6] Elsharkawy A.M., Yousef S.Kh., Hashem S. and Alikhan A.A.: "Compressibility Factor for Gas Condensates," paper SPE 59702 presented at the SPE Permian Basin Oil and Gas Recovery Conference, Midland, Texas, USA, March 21-23, 2000.

[7] Elsharkawy, A.M.: "Efficient Methods for Calculations of Compressibility, Density and Viscosity of Natural Gases," Fluid Phase Equilib., 218 (1), 2004, pp. 1-13.

[8] Elsharkawy, A.M. and Elkamel, A.: "Compressibility Factor for Sour Gas Reservoirs," paper SPE 64284 presented at the 2000 SPE Asia Pacific Oil and Gas Conference and Exhibition, Brisbane, Australia, October 16-18, 2000.

[9] Sutton, R.P.: "Fundamental PVT Calculations for Associated and Gas/Condensate Natural Gas Systems," paper SPE 97099 presented at the SPE Annual Technical Conference and Exhibition, Dallas, Texas, October 9-12, 2005, pp. 270-284. 
[10] Kesler, M.G. and Lee, B.I.: "Improve Prediction of Enthalpy of Fraction," Hyd. Proc., March, 1976, pp. 153-158.

[11] Whitson, C. H.: "Effect of C7+ Properties on Equationof-State Predictions," SPEJ, December, 1987, pp. 685696.

[12] Riazi, M.R. and Daubert, T.E.: "Characterization Parameters for Petroleum Fractions," Ind. Eng. Chem. Res., 26 (24), 1987, pp. 755-759.

[13] Wichert, E. and Aziz, K.: "Calculation of Z's for Sour Gases," Hyd. Proc., 51 (5), 1972, pp. 119-122.

[14] Standing, M.B.: "Volumetric and Phase Behavior of Oil Field Hydrocarbon Systems," $9^{\text {th }}$ ed., Society of Petroleum Engineers of AIME, Dallas, Texas, 1981.

[15] Lee, J. and Wattenbarger, R.A.: "Gas Reservoir Engineering," Society of Petroleum Engineers, Vol. 5, Richardson, Texas, USA, 1996.

[16] Hankinson, R.W., Thomas, L.K. and Phillips, K.A.: "Predict Natural Gas Properties," Hyd. Proc., 48, April, 1969, pp. 106-108.

[17] Hall, K.R. and Yarborough, L.: "A New Equation of State for Z-Factor Calculations," Oil \& Gas J., 71 (25), 1973, pp. 82-92.

[18] Dranchuk, P.M., Purvis, R.A. and Robinson, D.B.: "Computer Calculation of Natural Gas Compressibility Factors Using the Standing and Katz Correlation," Institute of Petroleum Technical Series, No. IP 74-008, 1974, pp. 1-13.

[19] Dranchuk, P.M. and Abou-Kassem, J.H.: "Calculation of Z factors for Natural Gases Using Equations of State," J. Can. Petrol. Technol., 14 (3), 1975, pp. 34-36.

[20] Hall, K.R. and Iglesias-Silva, G.A.: "Improved Equations for the Standing-Katz Tables," Hyd. Proc., 86 (4), 2007, pp. 107-110.

[21] Papay, J.: "A Termelestechnologiai Parameterek Valtozasa a Gaztelepek Muvelese Soran," OGIL Musz. Tud Kozl., Budapest, 1968, pp. 267-273.

[22] Beggs, D.H. and Brill, J.P.: "A Study of Two-Phase Flow in Inclined Pipes," J. Petrol. Technol., 25 (5), 1973, pp. 607-617.

[23] Gopal, V. N.: "Gas Z-Factor Equations Developed for Computer," Oil \& Gas J., August 8, 1977, pp. 58-60.

[24] Burnett, R.R.: "Calculator Gives Compressibility Factors," The Oil and Gas Journal, June 11, 1979, pp. 70-74.

[25] Kumar, N.: "Compressibility Factors for Natural and Sour Reservoir Gases by Correlations and Cubic Equations of State," MS Thesis, Texas Tech University, Lubbock, Tex, USA, 2004.

[26] Bahadori, A., Mokhatab, S. and Towler, B.F.: "Rapidly Estimating Natural Gas Compressibility Factor," J. Nat. Gas Chem., 16 (4), 2007, pp. 349-353.

[27] Al-Anazi, B.D. and Al-Quraishi, A.A.: "New Correlation for Z-Factor Using Genetic Programming Technique," paper SPE 128878 presented at the SPE Oil and Gas India Conference and Exhibition, Mumbai, India, January 20-22, 2010.
[28] Azizi, N., Behbahani, R. and Isazadeh, M.A.: "An Efficient Correlation for Calculating Compressibility Factor of Natural Gases," J. Nat. Gas Chem., 19 (6), 2010, pp. 642-645.

[29] Heidaryan E., Salarabadi A. and Moghadasi J.: “A Novel Correlation Approach for Prediction of Natural Gas Compressibility Factor," J. Nat. Gas Chem., 19, 2010, pp. 189-192.

[30] Heidaryan, E., Moghadasi, J. and Rahimi, M.: "New Correlations to Predict Natural Gas Viscosity and Compressibility Factor," J. Petrol. Sci. Eng., 73 (1-2), 2010, pp. 67-72.

[31] Shokir, E.M.E.-M., El-Awad, M.N., Al-Quraishi, A.A., AlMahdy, O.A.: "Compressibility Factor Model of Sweet, Sour, and Condensate Gases Using Genetic Programming," Chem. Eng. Res. Des., 90 (6), 2012, pp. 785-792.

[32] Sanjari, E. and Lay, E.N.: "An Accurate Empirical Correlation for Predicting Natural Gas Compressibility Factors," J. Nat. Gas Chem., 21 (2), 2012, pp. 184-188.

[33] Mahmoud, M.A.: "Development of a New Correlation of Gas Compressibility Factor (Z-Factor) for High Pressure Gas Reservoirs," paper SPE 164587 presented at the SPE North Africa Technical Conference and Exhibition, Cairo, Egypt, April 15-17, 2013.

[34] Obuba, J., Ikiesnkimama, S.S., Ubani, C.E. and Ekeke, I.C.: "Natural Gas Compressibility Factor Correlation Evaluation for Niger Delta Gas Fields," IOSR Journal of Electrical and Electronics Engineering, 6 (4), Jul. - Aug., 2013, pp. 1-10.

\section{Appendix A. Pseudo-critical pressure and temperature calculation methods}

Sutton Modification to SBV Mixing Rules (SSBV Mixing Rules) (1985)

SBV Mixing rules

$$
\begin{gathered}
J=\left(\frac{1}{3}\right)\left[\sum_{i=1}^{n} y_{i}\left(\frac{T_{c}}{P_{c}}\right)_{i}\right]+\left(\frac{2}{3}\right)\left[\sum_{i=1}^{n} y_{i}\left(\sqrt{\frac{T_{c}}{P_{c}}}\right)_{i}\right]^{2} \\
K=\sum_{i=1}^{n} y_{i}\left(\frac{T_{c}}{\sqrt{P_{c}}}\right)_{i}
\end{gathered}
$$

\section{Sutton Modification}

$$
\begin{gathered}
F_{J}=\left(\frac{1}{3}\right)\left[y\left(\frac{T_{c}}{P_{c}}\right)\right]_{C_{7+}}+\left(\frac{-}{3}\right)\left[y\left(\sqrt{\frac{T_{c}}{P_{c}}}\right)\right]_{C_{7+}}^{2} \\
E_{J}=0.6081 F_{J}+1.1325 F_{J}^{2}-14.004 F_{J} y_{C_{7+}}+ \\
64.434 F_{J} y_{C_{7+}}^{2} \\
E_{K}=\left(\sqrt{\frac{T_{c}}{P_{c}}}\right)_{C_{7+}}\left[0.3129 y_{C_{7+}}-4.8156 y_{C_{7+}}^{2} 27.3751 y_{C_{7+}}^{3}\right] \\
J^{\prime}=J-E_{J} \\
K^{\prime}=K-E_{K} \\
T_{p c}=K^{\prime 2} / J^{\prime} \\
P_{p c}=T_{p c} / J^{\prime} \\
\text { Corredor et al. Mixing Rules (1992) } \\
J=\alpha_{0}+\sum_{i=1}^{3} \alpha_{i} y_{i}\left(\frac{T_{c}}{P_{c}}\right)_{i}+\alpha_{4} \sum_{j=1}^{6} y_{j}\left(\frac{T_{c}}{P_{c}}\right)_{j}+ \\
\alpha_{5}\left[\sum_{j=1}^{6} y_{j}\left(\frac{T_{c}}{P_{c}}\right)_{j}\right]^{2}+\alpha_{6}\left(y_{C_{7+}} M_{C_{7+}}\right)+\alpha_{7}\left(y_{C_{7+}} M_{C_{7+}}\right)^{2}
\end{gathered}
$$




$$
\begin{gathered}
K=\beta_{0}+\sum_{i=1}^{3} \beta_{i} y_{i}\left(\frac{T_{c}}{\sqrt{P_{c}}}\right)_{i}+\beta_{4} \sum_{j=1}^{6} y_{j}\left(\frac{T_{c}}{\sqrt{P_{C}}}\right)_{j}+ \\
\beta_{5}\left[\sum_{j=1}^{6} y_{j}\left(\frac{T_{c}}{\sqrt{P_{c}}}\right)_{j}\right]^{2}+\beta_{6}\left(y_{C_{7+}} M_{C_{7+}}\right)+\beta_{7}\left(y_{C_{7+}} M_{C_{7+}}\right)^{2} \\
\text { Where } y_{i} \in\left\{y_{H_{2} S}, y_{C_{2}}, y_{N_{2}}\right\} \text { and } y_{j} \in\left\{y_{C_{1}}, y_{C_{2}}, \ldots, y_{C_{6}}\right\} \\
\text { respectively. }
\end{gathered}
$$

Piper et al. Mixing Rules (1993)

$$
\begin{aligned}
& J=\alpha_{0}+\sum_{i=1}^{3} \alpha_{i} y_{i}\left(\frac{T_{c}}{P_{c}}\right)_{i}+\alpha_{4} \sum_{j=1}^{6} y_{j}\left(\frac{T_{c}}{P_{c}}\right)_{j}+ \\
& \alpha_{5}\left[\sum_{j=1}^{6} y_{j}\left(\frac{T_{c}}{P_{c}}\right)_{j}\right]^{2}+\alpha_{6}\left(y_{C_{7+}} M_{C_{7+}}\right)+\alpha_{7}\left(y_{C_{7+}} M_{C_{7+}}\right)^{2} \\
& K=\beta_{0}+\sum_{i=1}^{3} \beta_{i} y_{i}\left(\frac{T_{c}}{\sqrt{P_{c}}}\right)_{i}+\beta_{4} \sum_{j=1}^{6} y_{j}\left(\frac{T_{c}}{\sqrt{P_{c}}}\right)_{j}+ \\
& \beta_{5}\left[\sum_{j=1}^{6} y_{j}\left(\frac{T_{c}}{\sqrt{P_{c}}}\right)_{j}\right]^{2}+\beta_{6}\left(y_{C_{7+}} M_{C_{7+}}\right)+\beta_{7}\left(y_{C_{7+}} M_{C_{7+}}\right)^{2} \\
& \text { Where } y_{i} \in\left\{y_{H_{2} S}, y_{C_{2}}, y_{N_{2}}\right\} \text { and } y_{j} \in\left\{y_{C_{1}}, y_{C_{2}}, \ldots, y_{C_{6}}\right\} \\
& \text { respectively. }
\end{aligned}
$$

Al-Sharkawy et al. Mixing Rules (2000)

$J=\sum_{i=1}^{6} y_{i}\left(\frac{T_{c}}{P_{c}}\right)_{i}+\left\{a_{0}+\left[a_{1}\left(y T_{c} / P_{c}\right)\right]_{C_{7+}}+\left[a_{2}\left(y T_{c} / P_{c}\right)\right]_{N_{2}}+\right.$

$$
\left.\left[a_{3}\left(y T_{c} / P_{c}\right)\right]_{C o_{2}}+\left[a_{4}\left(y T_{c} / P_{c}\right)\right]_{H_{2} S}\right\}
$$$$
K=\sum_{i=1}^{6} y_{i}\left(\frac{T_{c}}{\sqrt{P_{c}}}\right)_{i}+\left\{b_{0}+\left[b_{1}\left(y T_{c} / \sqrt{P_{c}}\right)\right]_{C_{7+}}+\right.
$$

$\left.\left[b_{2}\left(y T_{c} / \sqrt{P_{c}}\right)\right]_{N_{2}}+\left[b_{3}\left(y T_{c} / \sqrt{P_{c}}\right)\right]_{c_{o_{2}}}+\left[b_{4}\left(y T_{c} / \sqrt{P_{c}}\right)\right]_{H_{2} S}\right\}$

\section{Al-Sharkawy Mixing Parameters (2004)}

$$
\begin{gathered}
J_{\text {inf }}=\alpha_{0}+\sum_{i=1}^{3} \alpha_{i} y_{i}\left(\frac{T_{c}}{P_{c}}\right)_{i}+\alpha_{4} \sum_{j=1}^{6} y_{j}\left(\frac{T_{c}}{P_{c}}\right)_{j}+ \\
\alpha_{5}\left(y_{C_{7+}} M_{C_{C_{+}}}\right) \\
K_{\text {inf }}=\beta_{0}+\sum_{i=1}^{3} \beta_{i} y_{i}\left(\frac{T_{c}}{\sqrt{P_{c}}}\right)_{i}+\beta_{4} \sum_{j=1}^{6} y_{j}\left(\frac{T_{c}}{\sqrt{P_{c}}}\right)_{j}+ \\
\beta_{5}\left(y_{C_{7_{+}}} M_{C_{7+}}\right) \\
\text { Where } y_{i} \in\left\{y_{H_{2} S}, y_{C_{o_{2}}}, y_{N_{2}}\right\} \text { and } y_{j} \in\left\{y_{C_{1}}, y_{C_{2}}, \ldots, y_{C_{6}}\right\} \\
\text { respectively. }
\end{gathered}
$$

Sutton Correlations (1985)

$$
\begin{aligned}
& T_{p c_{H C}}=169.2+349.5\left(\gamma_{g_{H C}}\right)-74\left(\gamma_{g_{H C}}\right)^{2} \\
& P_{p c_{H C}}=756.8-131\left(\gamma_{g_{H C}}\right)-3.6\left(\gamma_{g_{H C}}\right)^{2}
\end{aligned}
$$

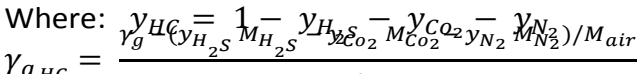

$$
\begin{aligned}
& \text { Piper et al. Mixing Rules (1993) } \\
& J=0.11582-0.45820 y_{H_{2} S} \frac{T_{c_{H_{2}} S}}{P_{c_{H_{2} S}}}-0.90348 y_{C_{O_{2}}} \frac{T_{c} C_{O_{2}}}{P_{c C_{o_{2}}}}- \\
& 0.66026 y_{N_{2}} \frac{T_{c_{N_{2}}}}{P_{c_{N_{2}}}}+0.70729\left(\gamma_{g}\right)-0.099397\left(\gamma_{g}\right)^{2} \\
& K=3.8216-0.06534 y_{H_{2} S} \frac{T_{c_{H_{2} S}}}{\sqrt{P_{c_{H_{2}} S}}}-0.42113 y_{\mathrm{Co}_{2}} \frac{T_{c}{C o_{2}}}{\sqrt{P_{c} c_{2}}}- \\
& 0.91249 y_{N_{2}} \frac{T_{c_{N_{2}}}}{\sqrt{P_{c_{N_{2}}}}}+17.438\left(\gamma_{g}\right)-3.2191\left(\gamma_{g}\right)^{2}
\end{aligned}
$$

\section{El-Sharkawy-El-Kamel Correlations (2000)}

$$
\begin{gathered}
T_{p c}=195.958+206.121\left(\gamma_{g}\right)+25.855\left(W_{H C}\right)- \\
6.421\left(W_{N H C}\right)+9.022\left(W_{H C}\right)^{2}+163.247\left(W_{N H C}\right)^{2} \\
P_{p c}=193.941-131.347\left(\gamma_{g}\right)+217.144\left(W_{H C}\right)+ \\
1060.349\left(W_{N H C}\right)+344.573\left(W_{H C}\right)^{2}-60.591\left(W_{N H C}\right)^{2} \\
\text { Sutton Correlations }(2005) \\
T_{p c_{H C}}=164.3+357.7\left(\gamma_{g_{H C}}\right)-67.7\left(\gamma_{g_{H C}}\right)^{2} \\
P_{p c_{H C}}=744-125.4\left(\gamma_{g_{H C}}\right)+5.9\left(\gamma_{g_{H C}}\right)^{2} \\
\text { Kesler and Lee Correlations }(\mathbf{1 9 7 6}) \\
T_{c}=341.7+811(\gamma)+(0.4244+0.1174(\gamma)) T_{b}+ \\
\quad(0.4669-3.2623(\gamma))\left(10^{5}\right) / T_{b} \\
P_{c}=\exp [8.3634-0.0566 / \gamma-(0.24244+ \\
\left.2.2898 / \gamma+0.11857 / \gamma^{2}\right)\left(10^{-3}\right) T_{b}+(1.4685+ \\
\left.3.648 / \gamma+0.47227 / \gamma^{2}\right)\left(10^{-7}\right) T_{b}^{2}-(0.42019+ \\
\left.\left.1.6977 / \gamma^{2}\right)\left(10^{-10}\right) T_{b}^{3}\right]
\end{gathered}
$$$$
\text { Whitson (1987) }
$$

$$
T_{b}=\left(4.5579(M)^{0.15178}(\gamma)^{0.15427}\right)^{3}
$$

\section{Riazi and Daubert Correlations (1987)}

$\theta=a(M)^{b}(\gamma)^{c} \exp [d(M)+e(\gamma)+f(M)(\gamma)]$

Wichert and Aziz Correction Method (1972) $\epsilon=120\left(A^{0.9}-A^{1.6}\right)+1.5\left(B^{0.5}-B^{4}\right)$

$$
\begin{gathered}
T_{p c}^{\prime}=T_{p c}-\epsilon \\
P_{p c}^{\prime}=\frac{P_{p c} T_{p c}^{\prime}}{\left[T_{p c}+B(1-B) \epsilon\right]} \\
A=y_{H_{2} S}+y_{C o_{2}} \\
B=y_{H_{2} S}
\end{gathered}
$$

Modified Wichert and Aziz Correction Method $\epsilon=107.6\left(A-A^{2.2}\right)+5.9\left(B^{0.06}-B^{0.68}\right)$

\section{Standing Correction Method}

$$
\begin{aligned}
& T_{p c}=y_{H C} T_{p c_{H C}}+y_{\mathrm{H}_{2} S} T_{c_{H_{2} S}}+y_{C_{O_{2}}} T_{c_{C O_{2}}}+y_{N_{2}} T_{c_{N_{2}}} \\
& P_{p c}=y_{H C} P_{p c_{H C}}+y_{H_{2} S} P_{c_{H_{2} S} S}+y_{C_{2}} P_{c_{C O_{2}}}+y_{N_{2}} P_{c_{N_{2}}}
\end{aligned}
$$

\section{Casey Correction Method}

$$
\begin{gathered}
T_{p c}^{\prime \prime}=\frac{T_{p c}^{\prime}\left(227.2 y_{N_{2}}+1165 y_{H_{2} O}\right)}{1-\left(y_{N_{2}}+y_{\mathrm{H}_{2} \mathrm{O}}\right)}-\left(246.1 y_{N_{2}}-400 y_{\mathrm{H}_{2} \mathrm{O}}\right) \\
P_{p c}^{\prime \prime}=\frac{P_{p c}^{\prime}\left(493.1 y_{N_{2}}+3200 y_{H_{2} O}\right)}{1-\left(y_{N_{2}}+y_{\mathrm{H}_{2} \mathrm{O}}\right)}-\left(162 y_{N_{2}}-1270 y_{\mathrm{H}_{2} \mathrm{O}}\right)
\end{gathered}
$$

The coefficients of corredor et al. mixing rules, Piper et al. mixing rules, Al-Sharkawy mixing rules, AlSharkawy mixing parameters and Riazi and Daubert correlations are shown in Table A-1 through Table A5 respectively.

\section{Papay Correlation (1968)}

$$
Z=1-\frac{3.53 P_{p r}}{10^{0.9813 T p r}}+\frac{0.274 P_{p r}^{2}}{10^{0.8157 T} p r}
$$

Beggs and Brill Z-Factor Correlation (1973)

$$
Z=A+\frac{1-A}{\exp (B)}+C P_{p r}^{D}
$$

Recommended range: $\quad 1.05<T_{p r}<2.0$

$$
P_{p r}<15
$$

Where:

$$
\begin{gathered}
A=1.39\left(T_{p r}-0.92\right)^{0.5}-0.36 T_{p r}-0.101 \\
B=\left(0.62-0.23 T_{p r}\right) P_{p r}+\left(\frac{0.066}{T_{p r}-0.86}-0.037\right) P_{p r}^{2}+\frac{0.32 P_{p r}^{6}}{10^{(9 T p r-9)}} \\
C=0.132-0.32 \log T_{p r} \\
D=10^{\left(0.3106-0.49 T_{p r}+0.1824 T_{p r}^{2}\right)}
\end{gathered}
$$

Gopal Method (1977)

$$
\begin{gathered}
Z=P_{p r}\left(0.711+3.66 T_{p r}\right)^{-1.4667}-1.6371\left(0.319 T_{p r}+\right. \\
0.522)+2.071
\end{gathered}
$$

Recommended range: $\quad 1.05 \leq T_{p r} \leq 3.0$

\section{Burnett Correlation (1979)}

$$
5.4 \leq P_{p r} \leq 15
$$

$$
Z=1+\left(Z^{\prime}-1\right)(\sin 90 U)^{N}
$$

Recommended range: $\quad 1.3 \leq T_{p r} \leq 1.85$

$$
P_{p r} \leq P_{p r}^{\prime}
$$

Where: $Z^{\prime}=0.3379 \ln \left(\ln \left(T_{p r}\right)\right)+1.091$

$$
\begin{gathered}
P_{p r}^{\prime}=21.46 Z^{\prime}-11.9 Z^{\prime 2}-5.9 \\
U=P_{p r} / P_{p r}^{\prime}
\end{gathered}
$$

$$
N=\left(1.1+0.26 T_{p r}+\left(1.04-1.42 T_{p r}\right) U\right)\left(\frac{\exp (U)}{T_{p r}}\right)
$$

Shell Oil Company (Kumar) Correlation (2004)

$Z=A+B P_{p r}+(1-A) \exp (-C)-D\left(\frac{P_{p r}}{10}\right)^{4}$

Where: $A=-0.101-0.36 T_{p r}+1.3868 \sqrt{T_{p r}-0.919}$

$$
\begin{gathered}
B=0.021+\frac{0.04275}{T_{p r}-0.65} \\
C=P_{p r}\left[E+F P_{p r}+G P_{p r}^{4}\right] \\
D=0.122 \exp \left[-11.3\left(T_{p r}-1\right)\right] \\
E=0.6222-0.224 T_{p r}
\end{gathered}
$$




$$
\begin{gathered}
F=\frac{0.0657}{T_{p r}-0.85}-0.037 \\
G=0.32 \exp \left[-19.53\left(T_{p r}-1\right)\right]
\end{gathered}
$$

Bahadori et al. Correlation (2007)

$Z=a+b P_{p r}+c P_{p r}^{2}+d P_{p r}^{3}$

Recommended range: $\quad 1.05 \leq T_{p r} \leq 2.4$

$0.2 \leq P_{p r} \leq 16$

Where: $a=A_{a}+B_{a} T_{p r}+C_{a} T_{p r}^{2}+D_{a} T_{p r}^{3}$

$b=A_{b}+B_{b} T_{p r}+C_{b} T_{p r}^{2}+D_{b} T_{p r}^{3}$

$c=A_{c}+B_{c} T_{p r}+C_{c} T_{p r}^{2}+D_{c} T_{p r}^{3}$

$d=A_{d}+B_{d} T_{p r}+C_{d} T_{p r}^{2}+D_{d} T_{p r}^{3}$

Al-Anazi and Al-Quraishi Correlation (2010)

$$
Z=\frac{2 E}{1.0482}+F
$$

Where: $A=-0.06708 P_{p r}+0.2360$

$$
\begin{gathered}
B=\frac{(3 A)^{2}-1.427}{T_{p r}}+0.9178 \\
C=B-(-2 A)(B)
\end{gathered}
$$

$$
\begin{gathered}
D=(-2 A)(B)(C) \\
E=\frac{C+D}{1.0474 T_{p r}}+0.9178 \\
F=\frac{D}{E^{2}}-E
\end{gathered}
$$

\section{Azizi et al. Correlation (2010)}

$$
Z=A+\frac{B+C}{D+E}
$$

Recommended range: $\quad 1.1 \leq T_{p r} \leq 2.0$

$$
0.2 \leq P_{p r} \leq 11
$$

Where: $A=a T_{p r}^{2.16}+b P_{p r}^{1.028}+c P_{p r}^{1.58} T_{p r}^{-2.1}+$

$$
d \ln \left(T_{p r}\right)^{-0.5}
$$

$$
B=e+f T_{p r}^{2.4}+g P_{p r}^{1.56}+h P_{p r}^{0.124} T_{p r}^{3.033}
$$

$C=i \ln \left(T_{p r}\right)^{-1.28}+j \ln \left(T_{p r}\right)^{1.37}+k \ln \left(P_{p r}\right)+$

$$
l \ln \left(P_{p r}\right)^{2}+m \ln \left(P_{p r}\right) \ln \left(T_{p r}\right)
$$

$$
D=1+n T_{p r}^{5.55}+o P_{p r}^{0.68} T_{p r}^{0.33}
$$

$E=p \ln \left(T_{p r}\right)^{1.18}+q \ln \left(T_{p r}\right)^{2.1}+r \ln \left(P_{p r}\right)+s \ln \left(P_{p r}\right)^{2}+$ $t \ln \left(P_{p r}\right) \ln \left(T_{p r}\right)$

Heidaryan-Salarabadi-Moghadasi Model (2010)

$$
Z=\frac{A_{1}+A_{2} \ln \left(P_{p r}\right)+A_{3}\left[\ln \left(P_{p r}\right)\right]^{2}+A_{4}\left[\ln \left(P_{p r}\right)\right]^{3}+\frac{A_{5}}{T_{p r}}+\frac{A_{6}}{T_{p r}^{2}}}{1+A_{7} \ln \left(P_{p r}\right)+A_{8}\left[\ln \left(P_{p r}\right)\right]^{2}+\frac{A_{9}}{T_{p r}}+\frac{A_{10}}{T_{p r}^{2}}}
$$

Recommended range: $\quad 1.2 \leq T_{p r} \leq 3.0$

$$
0.2 \leq P_{p r} \leq 15
$$

\section{Heidaryan-Moghadasi-Rahimi Model (2010)}

$Z=\ln \left(\frac{A_{1}+A_{3} \ln \left(P_{p r}\right)+\frac{A_{5}}{T_{p r}}+A_{7}\left[\ln \left(P_{p r}\right)\right]^{2}+\frac{A_{9}}{T_{p r}^{2}}+\frac{A_{11}}{T_{p r}} \ln \left(P_{p r}\right)}{1+A_{2} \ln \left(P_{p r}\right)+\frac{A_{4}}{T_{p r}}+A_{6}\left[\ln \left(P_{p r}\right)\right]^{2}+\frac{A_{8}}{T_{p r}^{2}}+\frac{A_{10}}{T_{p r}} \ln \left(P_{p r}\right)}\right)$

$$
\text { Recommended range: } \quad 1.2 \leq T_{p r} \leq 3.0
$$$$
0.2 \leq P_{p r} \leq 15
$$

For more accuracy, two sets of coefficients $A_{1}$ through $A_{11}$ values were determined by minimizing the residual sum of squares of this proposed correlation.

\section{Shokir et al. Correlation (2012)}

$$
\begin{gathered}
Z=A+B+C+D+E \\
\text { Where: } A=2.679562 \frac{\left(2 T_{p r}-P_{p r}-1\right)}{\left[\frac{\left(P_{p r}^{2}+T_{p r}^{3}\right)}{P_{p r}}\right]} \\
B=-7.686825\left[\frac{P_{p r} T_{p r}+P_{p r}^{2}}{T_{p r} P_{p r}+2 T_{p r}^{2}+T_{p r}^{3}}\right] \\
C=-0.000624\left[T_{p r}^{2} P_{p r}-T_{p r} P_{p r}^{2}+T_{p r} P_{p r}^{3}+2 T_{p r} P_{p r}-\right. \\
\left.2 P_{p r}^{2}+2 P_{p r}^{3}\right] \\
D=3.067747 \frac{\left(T_{p r}-P_{p r}\right)}{\left(P_{p r}^{2}+T_{p r}+P_{p r}\right)} \\
E=\frac{0.068059}{T_{p r} P_{p r}}+0.139489 T_{p r}^{2}+0.081873 P_{p r}^{2}-\frac{0.041098 T_{p r}}{P_{p r}}+ \\
\frac{8.152325 P_{p r}}{T_{p r}}-1.63028 P_{p r}+0.24287 T_{p r}-2.64988
\end{gathered}
$$

Sanjari and Nemati Lay Correlation (2012)

$$
Z=1+A_{1} P_{p r}+A_{2} P_{p r}^{2}+\frac{A_{3} P_{p r}^{A_{4}}}{T_{p r}^{A_{5}}}+\frac{A_{6} P_{p r}^{\left(A_{4}+1\right)}}{T_{p r}^{A_{7}}}+\frac{A_{8} P_{p r}^{\left(A_{4}+2\right)}}{T_{p r}^{\left(A_{7}+1\right)}}
$$

Recommended range: $\quad 1.01 \leq T_{p r} \leq 3.0$

$$
0.01 \leq P_{p r} \leq 15
$$

M.A. Mahmoud Correlation (2013)

$$
\begin{aligned}
Z=0.702 \exp ( & \left.-2.5 T_{p r}\right) P_{p r}^{2} \\
& -5.524 \exp \left(-2.5 T_{p r}\right) P_{p r} \\
& +0.044 T_{p r}^{2}-0.164 T_{p r}+1.15
\end{aligned}
$$

Niger Delta Correlation (2013)

$$
Z=6.41824-0.013363 P_{p r}-3.351293 T_{p r}
$$

The coefficients of Bahadori et al. correlation, Azizi et al. correlation, Heidaryan-Salarabadi-
Moghadasi model, Heidaryan-Moghadasi-Rahimi model and Sanjari and Nemati Lay correlation are shown in Table B-1 through Table B-5 respectively. 
Table A-1 Coefficients of Corredor et al. mixing rules

\begin{tabular}{|c|c|c|}
\hline $\boldsymbol{i}$ & $\alpha_{i}$ & $\boldsymbol{\beta}_{i}$ \\
\hline $\boldsymbol{O}$ & $1.5303 \mathrm{E}-01$ & $2.6662 \mathrm{E}+00$ \\
\hline 1 & 9.0991E-01 & 9.7778E-01 \\
\hline 2 & 9.5869E-01 & 9.7607E-01 \\
\hline 3 & $6.6612 E-01$ & $7.4161 \mathrm{E}-01$ \\
\hline 4 & 4.7920E-O1 & $5.2672 \mathrm{E}-\mathrm{O} 1$ \\
\hline 5 & $3.4198 E-01$ & $1.6886 \mathrm{E}-02$ \\
\hline 6 & $2.0370 E-02$ & $4.5333 \mathrm{E}-\mathrm{O} 1$ \\
\hline 7 & - 8.4700E-05 & $-3.1884 E-03$ \\
\hline
\end{tabular}

Table A-2 Coefficients of Piper et al. mixing rules

\begin{tabular}{|c|c|c|}
\hline $\boldsymbol{i}$ & $\boldsymbol{\alpha}_{\boldsymbol{i}}$ & $\boldsymbol{\beta}_{\boldsymbol{i}}$ \\
\hline $\boldsymbol{O}$ & $5.2073 \mathrm{E}-\mathrm{O2}$ & $-3.9741 \mathrm{E}-\mathrm{O1}$ \\
\hline $\boldsymbol{I}$ & $1.0160 \mathrm{E}+\mathrm{OO}$ & $1.0503 \mathrm{E}+\mathrm{OO}$ \\
\hline $\boldsymbol{Z}$ & $8.6961 \mathrm{E}-01$ & $9.6592 \mathrm{E}-\mathrm{O} 1$ \\
\hline 3 & $7.2646 \mathrm{E}-01$ & $7.8569 \mathrm{E}-01$ \\
\hline $\boldsymbol{4}$ & $8.5101 \mathrm{E}-01$ & $9.8211 \mathrm{E}-01$ \\
\hline $\boldsymbol{5}$ & 0.0 & 0.0 \\
\hline $\boldsymbol{6}$ & $2.0818 \mathrm{E}-02$ & $4.5536 \mathrm{E}-01$ \\
\hline $\boldsymbol{7}$ & $-1.5060 \mathrm{E}-04$ & $-3.7684 \mathrm{E}-03$ \\
\hline
\end{tabular}

Table A-3 Coefficients of Al-Sharkawy et al. mixing rules

\begin{tabular}{|c|c|c|}
\hline $\boldsymbol{i}$ & $\boldsymbol{a}_{i}$ & $\boldsymbol{b}_{i}$ \\
\hline $\boldsymbol{O}$ & -0.040279933 & -0.776423332 \\
\hline $\boldsymbol{1}$ & 0.881709332 & 1.030721752 \\
\hline 2 & 0.800591625 & 0.734009058 \\
\hline 3 & 1.037850321 & 0.909963446 \\
\hline 4 & 1.059063178 & 0.888959152 \\
\hline
\end{tabular}

Table A-4 Coefficients of Al-Sharkawy et al. mixing parameters.

\begin{tabular}{|c|c|c|}
\hline $\boldsymbol{i}$ & $\boldsymbol{\alpha}_{\boldsymbol{i}}$ & $\boldsymbol{\beta}_{\boldsymbol{i}}$ \\
\hline $\boldsymbol{o}$ & 0.036983 & -0.7765003 \\
\hline $\boldsymbol{1}$ & $\mathbf{1 . 0 4 3 9 0 2}$ & $\mathbf{1 . 0 6 9 5 3 1 7}$ \\
\hline $\boldsymbol{2}$ & 0.894942 & 0.9850308 \\
\hline 3 & 0.792231 & 0.8617653 \\
\hline $\boldsymbol{4}$ & 0.882295 & $\mathbf{1 . 0 1 2 7 0 5 4}$ \\
\hline $\mathbf{5}$ & 0.018637 & 0.4014645 \\
\hline
\end{tabular}

Table A-5 Coefficients of Riazi and Daubert correlations.

\begin{tabular}{|c|c|c|c|c|c|c|}
\hline $\boldsymbol{\theta}$ & $\boldsymbol{a}$ & $\boldsymbol{b}$ & $\boldsymbol{c}$ & $\boldsymbol{d}$ & $\boldsymbol{e}$ & $\boldsymbol{f}$ \\
\hline $\boldsymbol{T}_{\boldsymbol{c}}{ }^{\boldsymbol{o}} \boldsymbol{R}$ & 544.4 & 0.2998 & 1.0555 & $-1.3478^{*} 10^{-4}$ & -0.61641 & 0.0 \\
\hline $\boldsymbol{P}_{c}, \boldsymbol{p} \boldsymbol{p} \boldsymbol{i} \boldsymbol{\alpha}$ & $4.5203^{*} 10^{4}$ & -0.8063 & 1.6015 & $-1.8078^{*} 10^{-3}$ & -0.3084 & 0.0 \\
\hline $\boldsymbol{T}_{\boldsymbol{b}},{ }^{\boldsymbol{o}} \boldsymbol{R}$ & 6.77857 & 0.401673 & -1.58262 & $3.77409^{*} 10^{-3}$ & 2.984036 & $-4.25288^{*} 10^{-3}$ \\
\hline
\end{tabular}

Table B-1 Coefficients of Bahadori et al. empirical correlation.

\begin{tabular}{|c|c|c|c|c|}
\hline $\boldsymbol{i}$ & $\boldsymbol{A}_{\boldsymbol{i}}$ & $\boldsymbol{B}_{\boldsymbol{i}}$ & $\boldsymbol{C}_{\boldsymbol{i}}$ & \multicolumn{1}{c|}{$\boldsymbol{D}_{\boldsymbol{i}}$} \\
\hline $\boldsymbol{a}$ & 0.9694690 & -1.3492380 & 1.4439590 & -0.3686000 \\
\hline $\boldsymbol{b}$ & -0.1077830 & -0.1270130 & 0.1008280 & -0.0123190 \\
\hline $\boldsymbol{c}$ & 0.0184810 & 0.0523405 & -0.0506880 & 0.0108700 \\
\hline $\boldsymbol{d}$ & -0.0005840 & -0.0021460 & 0.0020961 & -0.0004590 \\
\hline
\end{tabular}

Table B-2 Coefficients of Azizi et al. empirical correlation.

\begin{tabular}{|c|c|c|c|}
\hline $\boldsymbol{a}$ & 0.0373142485385592 & $\boldsymbol{k}$ & -24449114791.1531 \\
\hline $\boldsymbol{b}$ & -0.0140807151485369 & $\boldsymbol{l}$ & 19357955749.3274 \\
\hline $\boldsymbol{c}$ & 0.0163263245387186 & $\boldsymbol{m}$ & -126354717916.607 \\
\hline $\boldsymbol{d}$ & 0.0307776478819813 & $\boldsymbol{n}$ & 623705678.385784 \\
\hline $\boldsymbol{e}$ & 13843575480.943800 & $\boldsymbol{o}$ & 17997651104.3330 \\
\hline $\boldsymbol{f}$ & -16799138540.763700 & $\boldsymbol{p}$ & 151211393445.064 \\
\hline $\boldsymbol{g}$ & 1624178942.6497600 & $\boldsymbol{q}$ & 139474437997.172 \\
\hline $\boldsymbol{h}$ & 13702270281.086900 & $\boldsymbol{r}$ & -24233012984.0950 \\
\hline $\boldsymbol{i}$ & -41645509.896474600 & $\boldsymbol{s}$ & 18938047327.5205 \\
\hline $\boldsymbol{j}$ & 237249967625.01300 & $\boldsymbol{t}$ & -141401620722.689 \\
\hline
\end{tabular}


Table B-3 Coefficients of Heidaryan-Salarabadi-Moghadasi model.

\begin{tabular}{|l|r|r|r|}
\hline$A_{1}$ & 1.11532372699824 & $A_{6}$ & 1.15753118672070 \\
\hline$A_{2}$ & -0.07903952088760 & $A_{7}$ & -0.05367780720737 \\
\hline$A_{3}$ & 0.01588138045027 & $A_{s}$ & 0.01465569989618 \\
\hline$A_{4}$ & 0.00886134496010 & $A_{9}$ & -1.80997374923296 \\
\hline$A_{5}$ & -2.16190792611599 & $A_{10}$ & 0.95486038773032 \\
\hline
\end{tabular}

Table B-4 Coefficients of Heidaryan-Moghadasi-Rahimi model.

\begin{tabular}{|c|c|c|}
\hline Coefficient & $0.2 \leq P_{p r} \leq 3$ & $3<P_{p r} \leq 15$ \\
\hline$A_{1}$ & $2.827793^{*} 10^{+00}$ & $3.252838 * 10^{+00}$ \\
\hline $\mathrm{A}_{2}$ & $-4.688191^{*} 10^{-01}$ & $-1.306424 * 10^{-01}$ \\
\hline$A_{3}$ & $-1.262288 * 10+\infty 0$ & $-6.449194 * 10^{-01}$ \\
\hline$A_{4}$ & $-1.536524 * 10^{+\infty}$ & $-1.518028 * 10^{+00}$ \\
\hline$A_{5}$ & $-4.535045^{*} 10^{+00}$ & $-5.391019 * 10+00$ \\
\hline$A_{6}$ & $6.895104^{*} 10^{-02}$ & $-1.379588 * 10^{-02}$ \\
\hline$A_{7}$ & $1.903869^{*} 10^{-01}$ & $6.600633^{*} 10^{-02}$ \\
\hline As & $6.200089^{*} 10^{-01}$ & $6.120783^{*} 10^{-01}$ \\
\hline A9 & $1.838479 * 10+00$ & $2.317431 * 10+00$ \\
\hline$A_{10}$ & $4.052367^{*} 10^{-01}$ & $1.632223^{*} 10^{-01}$ \\
\hline$A_{11}$ & $1.073574 * 10+00$ & $5.660595 * 10^{-01}$ \\
\hline
\end{tabular}

Table B-5 Coefficients of Sanjari and Nemati lay empirical correlation.

\begin{tabular}{|c|c|c|}
\hline Coefficient & $\mathbf{0 . 0 1} \leq \boldsymbol{P}_{\boldsymbol{p r}} \leq \mathbf{3 . 0}$ & $\mathbf{3 . 0} \leq \boldsymbol{P}_{\boldsymbol{p r}} \leq \mathbf{1 5}$ \\
\hline $\boldsymbol{A}_{\mathbf{1}}$ & 0.007698 & 0.015642 \\
\hline $\boldsymbol{A}_{\boldsymbol{1}}$ & 0.003839 & 0.000701 \\
\hline $\boldsymbol{A}_{\boldsymbol{4}}$ & -0.467212 & 2.341511 \\
\hline $\boldsymbol{A}_{\boldsymbol{5}}$ & 1.018801 & -0.657903 \\
\hline $\boldsymbol{A}_{\boldsymbol{6}}$ & 3.805723 & 8.902112 \\
\hline $\boldsymbol{A} \boldsymbol{A}$ & -0.087361 & -1.136000 \\
\hline $\boldsymbol{A}$ & 7.138305 & 3.543614 \\
\hline
\end{tabular}

Table C-1 statistical parameters values for explict emperical correlations when using SSBV, Whitson,Kesler\&Lee, Wichert\&Aziz and Caseymethod for ppe and tpe.

\begin{tabular}{|c|c|c|c|c|c|c|}
\hline Explicit empirical correlation & Year & RSS & RMSE & AARE $\%$ & SD & $\mathbf{R}^{\mathbf{2}}$ \\
\hline New proposed correlation & 2018 & 0.5361 & 0.0273 & 1.6484 & 2.2817 & 0.9578 \\
\hline Heidaryan-Moghadasi-Rahimi & 2010 & 0.6003 & 0.0289 & 1.6938 & 2.3927 & 0.9527 \\
\hline Shell 0il Company & 2004 & 0.6080 & 0.0290 & 1.7682 & 2.4726 & 0.9521 \\
\hline Heidaryan-Salarabadi-Moghadasi & 2010 & 0.7628 & 0.0325 & 2.1425 & 2.8275 & 0.9399 \\
\hline Beggs and Brill & 1973 & 0.7775 & 0.0330 & 2.2082 & 2.7293 & 0.9355 \\
\hline Sanjari and Nemati Lay & 2012 & 1.2079 & 0.0409 & 2.4920 & 3.2455 & 0.9048 \\
\hline Shokir et al. & 2012 & 9.9010 & 0.1172 & 7.2638 & 9.4227 & 0.2197 \\
\hline Azizi et al. & 2010 & 55.990 & 0.2787 & 23.096 & 23.516 & - \\
\hline M.A. Mahmoud & 2013 & 61.823 & 0.2928 & 12.466 & 21.314 & - \\
\hline Papay & 1968 & 92.456 & 0.3581 & 20.715 & 27.334 & - \\
\hline Gopal & 1977 & 103.38 & 0.3787 & 28.380 & 33.101 & - \\
\hline Bahadori et al. & 2007 & 866.48 & 1.0963 & 91.578 & 92.410 & - \\
\hline Niger Delta & 2013 & 972.80 & 1.1616 & 84.401 & 100.75 & - \\
\hline Al-Anazi and Al-Quraishi & 2010 & $6.0^{*} 10^{8}$ & $9.1 * 10^{2}$ & $5.2^{*} 10^{3}$ & $7.0^{*} 10^{4}$ & - \\
\hline Burnett & 1979 & - & - & - & - & - \\
\hline
\end{tabular}


Table C-2 statistical parameters values for explict emperical correlations when using SSBV, Whitson,Kesler\&Lee, modified Wichert\&Aziz and Caseymethod for ppe and tpe.

\begin{tabular}{|c|c|c|c|c|c|c|}
\hline Explicit empirical correlation & Year & RSS & RMSE & AARE $\%$ & SD & $\mathrm{R}^{\mathbf{2}}$ \\
\hline New proposed correlation & 2018 & 0.5418 & 0.0274 & 1.6735 & 2.2880 & 0.9573 \\
\hline Heidaryan-Moghadasi-Rahimi & 2010 & 0.6060 & 0.0289 & 1.6847 & 2.3939 & 0.9522 \\
\hline Shell 0il Company & 2004 & 0.6073 & 0.0290 & 1.7650 & 2.4597 & 0.9521 \\
\hline Heidaryan-Salarabadi-Moghadasi & 2010 & 0.7539 & 0.0323 & 2.1254 & 2.7986 & 0.9406 \\
\hline Beggs and Brill & 1973 & 0.7925 & 0.0334 & 2.2378 & 2.7532 & 0.9342 \\
\hline Sanjari and Nemati Lay & 2012 & 1.2218 & 0.0412 & 2.4845 & 3.2504 & 0.9037 \\
\hline Shokir et al. & 2012 & 9.6890 & 0.1159 & 7.2097 & 9.3335 & 0.2364 \\
\hline Azizi et al. & 2010 & 56.125 & 0.2790 & 23.125 & 23.547 & - \\
\hline M.A.Mahmoud & 2013 & 61.822 & 0.2928 & 12.479 & 21.311 & - \\
\hline Papay & 1968 & 92.005 & 0.3572 & 20.653 & 27.269 & - \\
\hline Gopal & 1977 & 102.62 & 0.3773 & 28.287 & 32.977 & - \\
\hline Bahadori et al. & 2007 & 863.70 & 1.0945 & 91.414 & 92.253 & - \\
\hline Niger Delta & 2013 & 960.09 & 1.1540 & 83.960 & 100.09 & - \\
\hline Al-Anazi and Al-Quraishi & 2010 & $2.2^{*} 10^{7}$ & $1.7^{*} 10^{2}$ & $1.6^{*} 10^{3}$ & $1.4 * 10^{4}$ & - \\
\hline Burnett & 1979 & - & - & - & - & - \\
\hline
\end{tabular}

Table C-3 statistical parameters values for explict emperical correlations when using SSBV, Riazi \&Daubert, Wichert\&Aziz and Casey method for ppe and tpe.

\begin{tabular}{|c|c|c|c|c|c|c|}
\hline Explicit empirical correlation & Year & RSS & RMSE & AARE $\%$ & SD & R $^{2}$ \\
\hline New proposed correlation & 2018 & 0.5404 & 0.0274 & 1.6537 & 2.2933 & 0.9574 \\
\hline Heidaryan-Moghadasi-Rahimi & 2010 & 0.5883 & 0.0286 & 1.6577 & 2.3760 & 0.9536 \\
\hline Shell 0il Company & 2004 & 0.5998 & 0.0288 & 1.7381 & 2.4666 & 0.9527 \\
\hline Beggs and Brill & 1973 & 0.7341 & 0.0321 & 2.1271 & 2.6586 & 0.9391 \\
\hline Heidaryan-Salarabadi-Moghadasi & 2010 & 0.7849 & 0.0330 & 2.2033 & 2.8739 & 0.9381 \\
\hline Sanjari and Nemati Lay & 2012 & 1.1962 & 0.0407 & 2.4386 & 3.2228 & 0.9057 \\
\hline Shokir et al. & 2012 & 10.098 & 0.1183 & 7.3428 & 9.5204 & 0.2042 \\
\hline Azizi et al. & 2010 & 55.597 & 0.2777 & 23.017 & 23.439 & - \\
\hline M.A. Mahmoud & 2013 & 64.298 & 0.2986 & 12.685 & 21.736 & - \\
\hline Papay & 1968 & 95.868 & 0.3646 & 21.057 & 27.823 & - \\
\hline Gopal & 1977 & 103.36 & 0.3786 & 28.367 & 33.100 & - \\
\hline Bahadori et al. & 2007 & 874.17 & 1.1011 & 91.951 & 92.799 & - \\
\hline Niger Delta & 2013 & 978.11 & 1.1647 & 84.695 & 101.02 & - \\
\hline Al-Anazi and Al-Quraishi & 2010 & $9.2^{*} 10^{10}$ & $1.1^{*} 10^{4}$ & $3.3^{*} 10^{4}$ & $8.2 * 10^{5}$ & - \\
\hline Burnett & 1979 & - & - & - & - & - \\
\hline
\end{tabular}

Table C-4 statistical parameters values for explict emperical correlations when using SSBV, Riazi \&Daubert, Modified Wichert\&Aziz and Casey method for ppe and tpe.

\begin{tabular}{|c|c|c|c|c|c|c|}
\hline Explicit empirical correlation & Year & RSS & RMSE & AARE $\%$ & SD & R $^{\mathbf{2}}$ \\
\hline New proposed correlation & 2018 & 0.5447 & 0.0275 & 1.6824 & 2.2965 & 0.9571 \\
\hline Heidaryan-Moghadasi-Rahimi & 2010 & 0.5927 & 0.0287 & 1.6517 & 2.3745 & 0.9533 \\
\hline Shell Oil Company & 2004 & 0.5976 & 0.0288 & 1.7410 & 2.4508 & 0.9529 \\
\hline Heidaryan-Salarabadi-Moghadasi & 2010 & 0.7749 & 0.0328 & 2.1851 & 2.8435 & 0.9389 \\
\hline Beggs and Brill & 1973 & 0.7480 & 0.0324 & 2.1524 & 2.6808 & 0.9379 \\
\hline Sanjari and Nemati Lay & 2012 & 1.2097 & 0.0410 & 2.4287 & 3.2268 & 0.9047 \\
\hline Shokir et al. & 2012 & 9.8844 & 0.1171 & 7.2889 & 9.4316 & 0.2210 \\
\hline Azizi et al. & 2010 & 55.732 & 0.2780 & 23.046 & 23.470 & - \\
\hline M.A. Mahmoud & 2013 & 64.304 & 0.2986 & 12.698 & 21.735 & - \\
\hline Papay & 1968 & 95.408 & 0.3638 & 20.994 & 27.758 & - \\
\hline Gopal & 1977 & 102.60 & 0.3772 & 28.274 & 32.976 & - \\
\hline Bahadori et al. & 2007 & 871.37 & 1.0993 & 91.785 & 92.642 & - \\
\hline Niger Delta & 2013 & 965.35 & 1.1571 & 84.251 & 100.36 & - \\
\hline Al-Anazi and Al-Quraishi & 2010 & $8.6^{*} 10^{9}$ & $3.4^{*} 10^{3}$ & $1.6^{*} 10^{4}$ & $2.7^{*} 10^{5}$ & - \\
\hline Burnett & 1979 & - & - & - & - & - \\
\hline
\end{tabular}


Table C-5 statistical parameters values for explict emperical correlations when using SSBV, Riazi \&Daubert, Lee, Wichert\&Aziz and Casey method for ppe and tpe.

\begin{tabular}{|c|c|c|c|c|c|c|}
\hline Explicit empirical correlation & Year & RSS & RMSE & AARE $\%$ & SD & R $^{\mathbf{2}}$ \\
\hline New proposed correlation & 2018 & 0.5356 & 0.0273 & 1.6464 & 2.2799 & 0.9578 \\
\hline Heidaryan-Moghadasi-Rahimi & 2010 & 0.6068 & 0.0290 & 1.7104 & 2.4032 & 0.9522 \\
\hline Shell Oil Company & 2004 & 0.6158 & 0.0292 & 1.7856 & 2.4845 & 0.9515 \\
\hline Heidaryan-Salarabadi-Moghadasi & 2010 & 0.7575 & 0.0324 & 2.1243 & 2.8163 & 0.9403 \\
\hline Beggs and Brill & 1973 & 0.7952 & 0.0334 & 2.2389 & 2.7569 & 0.9340 \\
\hline Sanjari and Nemati Lay & 2012 & 1.1965 & 0.0407 & 2.5099 & 3.2402 & 0.9057 \\
\hline Shokir et al. & 2012 & 9.7668 & 0.1164 & 7.2141 & 9.3599 & 0.2303 \\
\hline Azizi et al. & 2010 & 56.206 & 0.2792 & 23.139 & 23.556 & - \\
\hline M.A. Mahmoud & 2013 & 59.901 & 0.2882 & 12.299 & 20.985 & - \\
\hline Papay & 1968 & 90.502 & 0.3543 & 20.531 & 27.055 & - \\
\hline Gopal & 1977 & 103.82 & 0.3795 & 28.464 & 33.168 & - \\
\hline Bahadori et al. & 2007 & 863.64 & 1.0945 & 91.449 & 92.271 & - \\
\hline Niger Delta & 2013 & 976.26 & 1.1636 & 84.603 & 100.93 & - \\
\hline Al-Anazi and Al-Quraishi & 2010 & $3.6^{*} 10^{\mathbf{8}}$ & $7.1^{*} 10^{\mathbf{2}}$ & $3.4^{*} 10^{3}$ & $5.5^{*} 10^{4}$ & - \\
\hline Burnett & 1979 & - & - & - & - & - \\
\hline
\end{tabular}

Table C-6 statistical parameters values for explict emperical correlations when using SSBV, Riazi \&Daubert, Kesler \& Lee, Modified Wichert\&Aziz and Casey method for ppe and tpe.

\begin{tabular}{|c|c|c|c|c|c|c|}
\hline Explicit empirical correlation & Year & RSS & RMSE & AARE $\%$ & SD & R $^{\mathbf{2}}$ \\
\hline New proposed correlation & 2018 & 0.5416 & 0.0274 & 1.6731 & 2.2869 & 0.9573 \\
\hline Heidaryan-Moghadasi-Rahimi & 2010 & 0.6129 & 0.0291 & 1.7006 & 2.4051 & 0.9517 \\
\hline Shell Oil Company & 2004 & 0.6155 & 0.0292 & 1.7803 & 2.4723 & 0.9515 \\
\hline Heidaryan-Salarabadi-Moghadasi & 2010 & 0.7490 & 0.0322 & 2.1080 & 2.7879 & 0.9410 \\
\hline Beggs and Brill & 1973 & 0.8105 & 0.0337 & 2.2698 & 2.7809 & 0.9327 \\
\hline Sanjari and Nemati Lay & 2012 & 1.2100 & 0.0410 & 2.5031 & 3.2448 & 0.9046 \\
\hline Shokir et al. & 2012 & 9.5539 & 0.1151 & 7.1601 & 9.2699 & 0.2471 \\
\hline Azizi et al. & 2010 & 56.341 & 0.2795 & 23.168 & 23.587 & - \\
\hline M.A. Mahmoud & 2013 & 59.900 & 0.2882 & 12.312 & 20.983 & - \\
\hline Papay & 1968 & 90.059 & 0.3534 & 20.469 & 26.991 & - \\
\hline Gopal & 1977 & 103.06 & 0.3781 & 28.371 & 33.044 & - \\
\hline Bahadori et al. & 2007 & 860.85 & 1.0927 & 91.284 & 92.114 & - \\
\hline Niger Delta & 2013 & 963.53 & 1.1560 & 84.159 & 100.27 & - \\
\hline Al-Anazi and Al-Quraishi & 2010 & $1.0^{*} 10^{7}$ & $1.2 * 10^{2}$ & $1.2 * 10^{3}$ & $9.3^{*} 10^{3}$ & - \\
\hline Burnett & 1979 & - & - & - & & - \\
\hline
\end{tabular}

Table C-7 statistical parameters values for explict emperical correlations when using SSBV, Riazi \&Daubert, Kesler \& Lee, Corredor et al. mixing rules for ppe and tpe.

\begin{tabular}{|c|c|c|c|c|c|c|}
\hline Explicit empirical correlation & Year & RSS & RMSE & AARE $\%$ & SD & R $^{\mathbf{2}}$ \\
\hline New proposed correlation & 2018 & 0.5583 & 0.0278 & 1.7145 & 2.3462 & 0.9560 \\
\hline Heidaryan-Moghadasi-Rahimi & 2010 & 0.6206 & 0.0293 & 1.8531 & 2.5181 & 0.9511 \\
\hline Shell Oil Company & 2004 & 0.6550 & 0.0301 & 1.9205 & 2.6077 & 0.9484 \\
\hline Beggs and Brill & 1973 & 0.6552 & 0.0303 & 1.9139 & 2.4991 & 0.9456 \\
\hline Heidaryan-Salarabadi-Moghadasi & 2010 & 0.9267 & 0.0359 & 2.5484 & 3.2118 & 0.9270 \\
\hline Sanjari and Nemati Lay & 2012 & 1.0813 & 0.0387 & 2.4411 & 3.2006 & 0.9148 \\
\hline Shokir et al. & 2012 & 10.693 & 0.1218 & 7.4613 & 9.7395 & 0.1573 \\
\hline Azizi et al. & 2010 & 55.074 & 0.2764 & 22.912 & 23.312 & - \\
\hline M.A. Mahmoud & 2013 & 59.240 & 0.2866 & 12.214 & 20.925 & - \\
\hline Papay & 1968 & 94.110 & 0.3613 & 21.386 & 27.713 & - \\
\hline Gopal & 1977 & 107.85 & 0.3868 & 29.322 & 33.856 & - \\
\hline Bahadori et al. & 2007 & 892.23 & 1.1124 & 93.033 & 93.837 & - \\
\hline Niger Delta & 2013 & 1045.2 & 1.2040 & 88.044 & 104.25 & - \\
\hline Al-Anazi and Al-Quraishi & 2010 & $1.5^{*} 10^{13}$ & $1.5^{*} 10^{5}$ & $4.6^{*} 10^{5}$ & $1.1^{*} 10^{7}$ & - \\
\hline Burnett & 1979 & - & - & - & - & - \\
\hline & & & & &
\end{tabular}


Table C-8 statistical parameters values for explict emperical correlations when using piper et al. mixing rules for ppe and tpe.

\begin{tabular}{|c|c|c|c|c|c|c|}
\hline Explicit empirical correlation & Year & RSS & RMSE & AARE \% & SD & R $^{\mathbf{2}}$ \\
\hline New proposed correlation & 2018 & 0.5772 & 0.0283 & 1.7721 & 2.3847 & 0.9545 \\
\hline Heidaryan-Moghadasi-Rahimi & 2010 & 0.6448 & 0.0299 & 1.9131 & 2.5671 & 0.9492 \\
\hline Shell Oil Company & 2004 & 0.6698 & 0.0305 & 1.9620 & 2.6276 & 0.9472 \\
\hline Beggs and Brill & 1973 & 0.6764 & 0.0308 & 1.9480 & 2.5360 & 0.9439 \\
\hline Heidaryan-Salarabadi-Moghadasi & 2010 & 0.9489 & 0.0363 & 2.5803 & 3.2522 & 0.9252 \\
\hline Sanjari and Nemati Lay & 2012 & 1.0750 & 0.0386 & 2.4648 & 3.2084 & 0.9153 \\
\hline Shokir et al. & 2012 & 10.587 & 0.1212 & 7.4673 & 9.7043 & 0.1657 \\
\hline Azizi et al. & 2010 & 55.111 & 0.2765 & 22.921 & 23.318 & - \\
\hline M.A. Mahmoud & 2013 & 58.121 & 0.2839 & 12.223 & 20.775 & - \\
\hline Papay & 1968 & 93.360 & 0.3598 & 21.464 & 27.661 & - \\
\hline Gopal & 1977 & 106.13 & 0.3837 & 29.072 & 33.589 & - \\
\hline Bahadori et al. & 2007 & 891.60 & 1.1120 & 93.027 & 93.829 & - \\
\hline Niger Delta & 2013 & 1024.4 & 1.1920 & 87.102 & 103.19 & - \\
\hline Al-Anazi and Al-Quraishi & 2010 & $7.0^{*} 10^{11}$ & $9.9^{*} 10^{3}$ & $3.3^{*} 10^{4}$ & $7.6 * 10^{5}$ & - \\
\hline Burnett & 1979 & - & - & - & - & - \\
\hline
\end{tabular}

Table C-9 statistical parameters values for explict emperical correlations when using Al-Sharkawy et al., Whitson and Kesler \& Leemethod for ppe and tpe.

\begin{tabular}{|c|c|c|c|c|c|c|}
\hline Explicit empirical correlation & Year & RSS & RMSE & AARE $\%$ & SD & R $^{2}$ \\
\hline New proposed correlation & 2018 & 0.5781 & 0.0283 & 1.7583 & 2.3845 & 0.9544 \\
\hline Heidaryan-Moghadasi-Rahimi & 2010 & 0.6417 & 0.0298 & 1.8426 & 2.5561 & 0.9494 \\
\hline Shell Oil Company & 2004 & 0.6792 & 0.0307 & 1.9395 & 2.6953 & 0.9465 \\
\hline Beggs and Brill & 1973 & 0.6599 & 0.0304 & 1.9271 & 2.5208 & 0.9452 \\
\hline Heidaryan-Salarabadi-Moghadasi & 2010 & 0.9426 & 0.0362 & 2.5646 & 3.2319 & 0.9257 \\
\hline Sanjari and Nemati Lay & 2012 & 1.1626 & 0.0402 & 2.5012 & 3.3092 & 0.9084 \\
\hline Shokir et al. & 2012 & 10.306 & 0.1196 & 7.2220 & 9.5631 & 0.1878 \\
\hline Azizi et al. & 2010 & 55.643 & 0.2778 & 22.991 & 23.407 & - \\
\hline M.A. Mahmoud & 2013 & 59.679 & 0.2877 & 11.847 & 20.845 & - \\
\hline Papay & 1968 & 94.108 & 0.3613 & 20.498 & 27.486 & - \\
\hline Gopal & 1977 & 122.42 & 0.4121 & 31.425 & 36.035 & \\
\hline Bahadori et al. & 2007 & 887.58 & 1.1095 & 92.697 & 93.534 & - \\
\hline Niger Delta & 2013 & 1200.2 & 1.2902 & 95.138 & 111.72 & \\
\hline Al-Anazi and Al-Quraishi & 2010 & $4.1^{*} 10^{8}$ & $7.5^{*} 10^{2}$ & $4.7^{*} 10^{3}$ & $5.8^{*} 10^{4}$ & - \\
\hline Burnett & 1979 & - & - & & & - \\
\hline
\end{tabular}

Table C-10 statistical parameters values for explict emperical correlations when using Al-Sharkawy et al and riazi \& Daubert method for ppe and tpe.

\begin{tabular}{|c|c|c|c|c|c|c|}
\hline Explicit empirical correlation & Year & RSS & RMSE & AARE $\%$ & SD & R $^{2}$ \\
\hline New proposed correlation & 2018 & 0.6304 & 0.0296 & 1.8722 & 2.4925 & 0.9503 \\
\hline Beggs and Brill & 1973 & 0.6288 & 0.0297 & 1.8606 & 2.4755 & 0.9478 \\
\hline Heidaryan-Moghadasi-Rahimi & 2010 & 0.6650 & 0.0304 & 1.9318 & 2.6098 & 0.9476 \\
\hline Shell Oil Company & 2004 & 0.7201 & 0.0316 & 2.0525 & 2.7839 & 0.9432 \\
\hline Heidaryan-Salarabadi-Moghadasi & 2010 & 1.0101 & 0.0374 & 2.6876 & 3.3460 & 0.9204 \\
\hline Sanjari and Nemati Lay & 2012 & 1.1652 & 0.0402 & 2.4637 & 3.3116 & 0.9082 \\
\hline Shokir et al. & 2012 & 10.656 & 0.1216 & 7.3520 & 9.7294 & 0.1602 \\
\hline Azizi et al. & 2010 & 54.980 & 0.2761 & 22.856 & 23.275 & - \\
\hline M.A. Mahmoud & 2013 & 63.246 & 0.2962 & 12.156 & 21.466 & - \\
\hline Papay & 1968 & 99.702 & 0.3719 & 21.039 & 28.280 & - \\
\hline Gopal & 1977 & 122.59 & 0.4123 & 31.443 & 36.063 & - \\
\hline Bahadori et al. & 2007 & 901.29 & 1.1181 & 93.351 & 94.221 & - \\
\hline Niger Delta & 2013 & 1214.6 & 1.2979 & 95.871 & 112.37 & - \\
\hline Al-Anazi and Al-Quraishi & 2010 & $1.8^{*} 10^{8}$ & $5.0^{*} 10^{2}$ & $3.7^{*} 10^{3}$ & $3.9^{*} 10^{4}$ & - \\
\hline Burnett & 1979 & - & - & - & & - \\
\hline
\end{tabular}


Table C-11 statistical parameters values for explict emperical correlations when using Al-Sharkawy et al, riazi \& Daubert and Daubert and kesler\& Lee method for ppe and tpe.

\begin{tabular}{|c|c|c|c|c|c|c|}
\hline Explicit empirical correlation & Year & RSS & RMSE & AARE \% & SD & R $^{\mathbf{2}}$ \\
\hline New proposed correlation & 2018 & 0.5574 & 0.0278 & 1.7070 & 2.3424 & 0.9561 \\
\hline Heidaryan-Moghadasi-Rahimi & 2010 & 0.6371 & 0.0297 & 1.8164 & 2.5462 & 0.9498 \\
\hline Shell Oil Company & 2004 & 0.6706 & 0.0305 & 1.9142 & 2.6746 & 0.9471 \\
\hline Beggs and Brill & 1973 & 0.6804 & 0.0309 & 1.9635 & 2.5528 & 0.9435 \\
\hline Heidaryan-Salarabadi-Moghadasi & 2010 & 0.9180 & 0.0357 & 2.5144 & 3.1932 & 0.9277 \\
\hline Sanjari and Nemati Lay & 2012 & 1.1521 & 0.0400 & 2.5300 & 3.3065 & 0.9092 \\
\hline Shokir et al. & 2012 & 10.120 & 0.1185 & 7.1543 & 9.4762 & 0.2025 \\
\hline Azizi et al. & 2010 & 56.018 & 0.2787 & 23.065 & 23.478 & - \\
\hline M.A. Mahmoud & 2013 & 56.894 & 0.2809 & 11.604 & 20.354 & - \\
\hline Papay & 1968 & 90.599 & 0.3545 & 20.177 & 26.985 & - \\
\hline Gopal & 1977 & 122.87 & 0.4128 & 31.499 & 36.096 & - \\
\hline Bahadori et al. & 2007 & 881.12 & 1.1055 & 92.407 & 93.221 & - \\
\hline Niger Delta & 2013 & 1200.5 & 1.2904 & 95.150 & 111.73 & - \\
\hline Al-Anazi and Al-Quraishi & 2010 & $4.4^{*} 10^{12}$ & $7.9^{*} 10^{4}$ & $2.3^{*} 10^{5}$ & $6.2^{*} 10^{6}$ & - \\
\hline Burnett & 1979 & - & - & - & - & - \\
\hline
\end{tabular}

Table C-12 statistical parameters values for explict emperical correlations when using Al-Sharkawy mixing parameters for ppe and tpe.

\begin{tabular}{|c|c|c|c|c|c|c|}
\hline Explicit empirical correlation & Year & RSS & RMSE & AARE \% & SD & R $^{\mathbf{2}}$ \\
\hline New proposed correlation & 2018 & 0.5755 & 0.0283 & 1.7661 & 2.3830 & 0.9546 \\
\hline Heidaryan-Moghadasi-Rahimi & 2010 & 0.6484 & 0.0300 & 1.9136 & 2.5754 & 0.9489 \\
\hline Shell Oil Company & 2004 & 0.6811 & 0.0307 & 1.9788 & 2.6514 & 0.9463 \\
\hline Beggs and Brill & 1973 & 0.6818 & 0.0309 & 1.9555 & 2.5463 & 0.9434 \\
\hline Heidaryan-Salarabadi-Moghadasi & 2010 & 0.9459 & 0.0362 & 2.5746 & 3.2502 & 0.9255 \\
\hline Sanjari and Nemati Lay & 2012 & 1.0904 & 0.0389 & 2.4833 & 3.2314 & 0.9141 \\
\hline Shokir et al. & 2012 & 10.416 & 0.1202 & 7.4037 & 9.6283 & 0.1791 \\
\hline Azizi et al. & 2010 & 55.349 & 0.2771 & 22.962 & 23.360 & - \\
\hline M.A. Mahmoud & 2013 & 56.757 & 0.2806 & 11.975 & 20.491 & - \\
\hline Papay & 1968 & 91.438 & 0.3561 & 21.187 & 27.353 & - \\
\hline Gopal & 1977 & 108.17 & 0.3873 & 29.444 & 33.905 & \\
\hline Bahadori et al. & 2007 & 889.49 & 1.1107 & 92.934 & 93.729 & - \\
\hline Niger Delta & 2013 & 1046.2 & 1.2046 & 88.331 & 104.29 & - \\
\hline Al-Anazi and Al-Quraishi & 2010 & $4.3^{*} 10^{14}$ & $7.7^{*} 10^{5}$ & $2.3^{*} 10^{6}$ & $6.3^{*} 10^{7}$ & - \\
\hline Burnett & 1979 & - & - & - & - & - \\
\hline
\end{tabular}

Table C-13 statistical parameters values for explict emperical correlations when using Sutton(1985), Standing, Wichert\& Aziz and Casey method for ppe and tpe.

\begin{tabular}{|c|c|c|c|c|c|c|}
\hline Explicit empirical correlation & Year & RSS & RMSE & AARE $\%$ & SD & R $^{\mathbf{2}}$ \\
\hline New proposed correlation & 2018 & 0.5346 & 0.0272 & 1.7200 & 2.2819 & 0.9579 \\
\hline Shell Oil Company & 2004 & 0.6280 & 0.0295 & 1.8617 & 2.5175 & 0.9505 \\
\hline Heidaryan-Moghadasi-Rahimi & 2010 & 0.6591 & 0.0302 & 1.8920 & 2.5707 & 0.9481 \\
\hline Beggs and Brill & 1973 & 0.7836 & 0.0331 & 2.1572 & 2.7101 & 0.9350 \\
\hline Heidaryan-Salarabadi-Moghadasi & 2010 & 0.8663 & 0.0347 & 2.3905 & 3.1048 & 0.9317 \\
\hline Sanjari and Nemati Lay & 2012 & 1.1245 & 0.0395 & 2.6121 & 3.2902 & 0.9114 \\
\hline Shokir et al. & 2012 & 9.6475 & 0.1157 & 7.1114 & 9.2906 & 0.2397 \\
\hline M.A. Mahmoud & 2013 & 51.473 & 0.2672 & 11.528 & 19.523 & \\
\hline Azizi et al. & 2010 & 56.764 & 0.2806 & 23.245 & 23.643 & - \\
\hline Papay & 1968 & 84.708 & 0.3428 & 20.277 & 26.295 & - \\
\hline Gopal & 1977 & 108.95 & 0.3887 & 29.498 & 34.005 & - \\
\hline Bahadori et al. & 2007 & 864.65 & 1.0951 & 91.695 & 92.446 & - \\
\hline Niger Delta & 2013 & 1025.4 & 1.1925 & 87.019 & 103.19 & - \\
\hline Al-Anazi and Al-Quraishi & 2010 & $1.5^{*} 10^{9}$ & $1.4^{*} 10^{3}$ & $8.9^{*} 10^{3}$ & $1.1^{*} 10^{5}$ & - \\
\hline Burnett & 1979 & - & - & - & - & - \\
\hline
\end{tabular}


Table C-14 statistical parameters values for explict emperical correlations when using Sutton(1985), Standing, Modified Wichert\& Aziz and Casey method for ppe and tpe.

\begin{tabular}{|c|c|c|c|c|c|c|}
\hline Explicit empirical correlation & Year & RSS & RMSE & AARE \% & SD & R $^{2}$ \\
\hline New proposed correlation & 2018 & 0.5449 & 0.0275 & 1.7516 & 2.2936 & 0.9571 \\
\hline Shell Oil Company & 2004 & 0.6302 & 0.0296 & 1.8609 & 2.5048 & 0.9503 \\
\hline Heidaryan-Moghadasi-Rahimi & 2010 & 0.6687 & 0.0305 & 1.9089 & 2.5743 & 0.9473 \\
\hline Beggs and Brill & 1973 & 0.8002 & 0.0335 & 2.1772 & 2.7347 & 0.9336 \\
\hline Heidaryan-Salarabadi-Moghadasi & 2010 & 0.8591 & 0.0345 & 2.3789 & 3.0765 & 0.9323 \\
\hline Sanjari and Nemati Lay & 2012 & 1.1369 & 0.0397 & 2.6056 & 3.2913 & 0.9104 \\
\hline Shokir et al. & 2012 & 9.3929 & 0.1141 & 7.0584 & 9.1841 & 0.2598 \\
\hline M.A. Mahmoud & 2013 & 51.473 & 0.2672 & 11.538 & 19.520 & - \\
\hline Azizi et al. & 2010 & 56.905 & 0.2809 & 23.276 & 23.674 & - \\
\hline Papay & 1968 & 84.323 & 0.3420 & 20.214 & 26.236 & - \\
\hline Gopal & 1977 & 108.14 & 0.3873 & 29.396 & 33.876 & - \\
\hline Bahadori et al. & 2007 & 861.87 & 1.0933 & 91.528 & 92.290 & - \\
\hline Niger Delta & 2013 & 1011.7 & 1.1846 & 86.512 & 102.50 & - \\
\hline Al-Anazi and Al-Quraishi & 2010 & $8.0^{*} 10^{7}$ & $3.3^{*} 10^{2}$ & $2.3^{*} 10^{3}$ & $2.6^{*} 10^{4}$ & - \\
\hline Burnett & 1979 & - & - & - & & - \\
\hline
\end{tabular}

Table C-15 statistical parameters values for explict emperical correlations when using piper et al. mixing rules for ppe and tpe.

\begin{tabular}{|c|c|c|c|c|c|c|}
\hline Explicit empirical correlation & Year & RSS & RMSE & AARE \% & SD & R $^{\mathbf{2}}$ \\
\hline New proposed correlation & 2018 & 0.4897 & 0.0261 & 1.7048 & 2.1754 & 0.9614 \\
\hline Shell Oil Company & 2004 & 0.5237 & 0.0270 & 1.7305 & 2.2674 & 0.9587 \\
\hline Heidaryan-Moghadasi-Rahimi & 2010 & 0.6270 & 0.0295 & 1.8935 & 2.5181 & 0.9506 \\
\hline Beggs and Brill & 1973 & 0.7555 & 0.0326 & 2.1500 & 2.6717 & 0.9373 \\
\hline Heidaryan-Salarabadi-Moghadasi & 2010 & 0.8049 & 0.0334 & 2.3067 & 3.0170 & 0.9366 \\
\hline Sanjari and Nemati Lay & 2012 & 1.1904 & 0.0406 & 2.6123 & 3.3559 & 0.9062 \\
\hline Shokir et al. & 2012 & 9.8669 & 0.1170 & 7.2581 & 9.4105 & 0.2224 \\
\hline Azizi et al. & 2010 & 56.433 & 0.2798 & 23.200 & 23.617 & - \\
\hline M.A. Mahmoud & 2013 & 59.221 & 0.2866 & 12.321 & 20.979 & - \\
\hline Papay & 1968 & 89.906 & 0.3531 & 20.952 & 27.101 & - \\
\hline Gopal & 1977 & 98.560 & 0.3697 & 27.926 & 32.417 & - \\
\hline Bahadori et al. & 2007 & 857.44 & 1.0905 & 91.303 & 92.039 & - \\
\hline Niger Delta & 2013 & 886.97 & 1.1091 & 80.461 & 95.989 & - \\
\hline Al-Anazi and Al-Quraishi & 2010 & $1.0^{*} 10^{11}$ & $1.2 * 10^{4}$ & $5.2^{*} 10^{4}$ & $9.3^{*} 10^{5}$ & - \\
\hline Burnett & 1979 & - & - & - & - & - \\
\hline
\end{tabular}

Table C-16 statistical parameters values for explict emperical correlations when using Al-Sharkawy- El Kamel emprical correlations for ppe and tpe.

\begin{tabular}{|c|c|c|c|c|c|c|}
\hline Explicit empirical correlation & Year & RSS & RMSE & AARE \% & SD & R $^{\mathbf{2}}$ \\
\hline Heidaryan-Salarabadi-Moghadasi & 2010 & 1.1004 & 0.0391 & 2.6006 & 3.2640 & 0.9133 \\
\hline New proposed correlation & 2018 & 1.6940 & 0.0485 & 3.3294 & 4.0378 & 0.8665 \\
\hline Shell Oil Company & 2004 & 1.7669 & 0.0495 & 3.4333 & 4.0495 & 0.8607 \\
\hline Heidaryan-Moghadasi-Rahimi & 2010 & 1.8167 & 0.0502 & 3.5252 & 4.1279 & 0.8568 \\
\hline Sanjari and Nemati Lay & 2012 & 2.5885 & 0.0599 & 4.2765 & 4.8969 & 0.7960 \\
\hline Beggs and Brill & 1973 & 2.8051 & 0.0628 & 4.5465 & 5.2090 & 0.7663 \\
\hline Shokir et al. & 2012 & 6.6353 & 0.0959 & 6.1721 & 7.8839 & 0.4771 \\
\hline M.A. Mahmoud & 2013 & 46.433 & 0.2538 & 11.172 & 18.546 & - \\
\hline Azizi et al. & 2010 & 62.932 & 0.2954 & 24.483 & 24.948 & - \\
\hline Papay & 1968 & 65.555 & 0.3015 & 17.260 & 22.941 & - \\
\hline Gopal & 1977 & 75.580 & 0.3238 & 24.658 & 28.421 & - \\
\hline Niger Delta & 2007 & 510.53 & 0.8415 & 61.992 & 72.763 & - \\
\hline Bahadori et al. & 2013 & 734.92 & 1.0096 & 84.482 & 85.245 & - \\
\hline Al-Anazi and Al-Quraishi & 2010 & $9.5 * 10^{5}$ & 36.308 & 313.43 & 2729.8 & - \\
\hline Burnett & 1979 & - & - & - & - & - \\
\hline
\end{tabular}


Table C-17 statistical parameters values for explict emperical correlations when using Sutton(1985), Standing, Wichert\& Aziz and Casey method for ppe and tpe.

\begin{tabular}{|c|c|c|c|c|c|c|}
\hline Explicit empirical correlation & Year & RSS & RMSE & AARE \% & SD & R $^{\mathbf{2}}$ \\
\hline New proposed correlation & 2018 & 0.5360 & 0.0273 & 1.7406 & 2.2824 & 0.9578 \\
\hline Shell Oil Company & 2004 & 0.5937 & 0.0287 & 1.7793 & 2.4241 & 0.9532 \\
\hline Heidaryan-Moghadasi-Rahimi & 2010 & 0.6312 & 0.0296 & 1.8721 & 2.5007 & 0.9503 \\
\hline Beggs and Brill & 1973 & 0.7573 & 0.0326 & 2.1316 & 2.6772 & 0.9371 \\
\hline Heidaryan-Salarabadi-Moghadasi & 2010 & 0.8367 & 0.0341 & 2.3412 & 3.0333 & 0.9341 \\
\hline Sanjari and Nemati Lay & 2012 & 1.1728 & 0.0403 & 2.5396 & 3.2726 & 0.9076 \\
\hline Shokir et al. & 2012 & 10.561 & 0.1210 & 7.5369 & 9.7284 & 0.1677 \\
\hline Azizi et al. & 2010 & 55.427 & 0.2773 & 22.996 & 23.410 & - \\
\hline M.A. Mahmoud & 2013 & 61.828 & 0.2928 & 12.727 & 21.443 & - \\
\hline Papay & 1968 & 93.131 & 0.3594 & 21.392 & 27.607 & - \\
\hline Gopal & 1977 & 97.851 & 0.3684 & 27.510 & 32.244 & - \\
\hline Bahadori et al. & 2007 & 871.10 & 1.0992 & 91.983 & 92.761 & - \\
\hline Niger Delta & 2013 & 909.73 & 1.1233 & 80.822 & 97.251 & - \\
\hline Al-Anazi and Al-Quraishi & 2010 & $2.0^{*} 10^{11}$ & $1.7^{*} 10^{4}$ & $5.4^{*} 10^{4}$ & $1.3^{*} 10^{5}$ & - \\
\hline Burnett & 1979 & - & - & - & & - \\
\hline
\end{tabular}

Table C-18 statistical parameters values for explict emperical correlations when using Sutton(1985), Standing, Modified Wichert\& Aziz and Casey method for ppe and tpe.

\begin{tabular}{|c|c|c|c|c|c|c|}
\hline Explicit empirical correlation & Year & RSS & RMSE & AARE \% & SD & R $^{\mathbf{2}}$ \\
\hline New proposed correlation & 2018 & 0.5474 & 0.0276 & 1.7756 & 2.2980 & 0.9569 \\
\hline Shell Oil Company & 2004 & 0.5984 & 0.0288 & 1.7849 & 2.4188 & 0.9528 \\
\hline Heidaryan-Moghadasi-Rahimi & 2010 & 0.6415 & 0.0298 & 1.9014 & 2.5073 & 0.9494 \\
\hline Beggs and Brill & 1973 & 0.7763 & 0.0330 & 2.1564 & 2.7078 & 0.9356 \\
\hline Heidaryan-Salarabadi-Moghadasi & 2010 & 0.8303 & 0.0339 & 2.3301 & 3.0070 & 0.9346 \\
\hline Sanjari and Nemati Lay & 2012 & 1.1887 & 0.0406 & 2.5347 & 3.2786 & 0.9063 \\
\hline Shokir et al. & 2012 & 10.317 & 0.1196 & 7.4785 & 9.6302 & 0.1870 \\
\hline Azizi et al. & 2010 & 55.560 & 0.2776 & 23.024 & 23.440 & - \\
\hline M.A. Mahmoud & 2013 & 61.794 & 0.2928 & 12.735 & 21.435 & - \\
\hline Papay & 1968 & 92.703 & 0.3586 & 21.323 & 27.546 & - \\
\hline Gopal & 1977 & 97.096 & 0.3670 & 27.414 & 32.117 & - \\
\hline Bahadori et al. & 2007 & 868.35 & 1.0974 & 91.818 & 92.606 & - \\
\hline Niger Delta & 2013 & 897.24 & 1.1155 & 80.379 & 96.580 & - \\
\hline Al-Anazi and Al-Quraishi & 2010 & $9.3^{*} 10^{9}$ & $3.6 * 10^{3}$ & $1.3^{*} 10^{4}$ & $2.8^{*} 10^{5}$ & - \\
\hline Burnett & 1979 & - & - & - & - & - \\
\hline
\end{tabular}

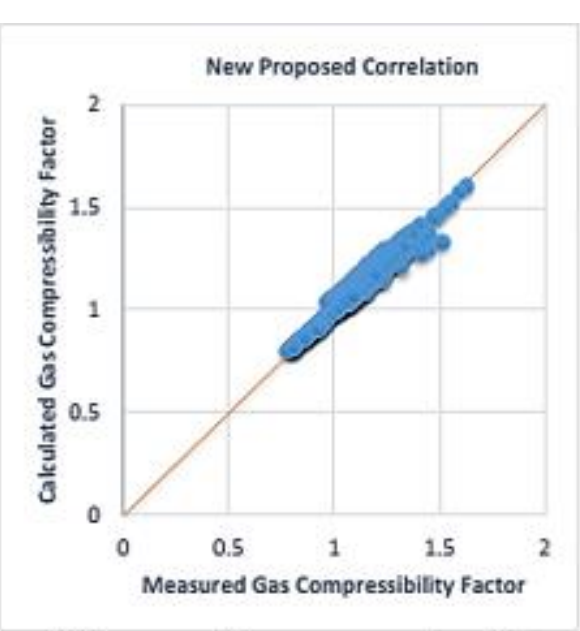

Figure C-1 Accuracy of the new proposed explicit

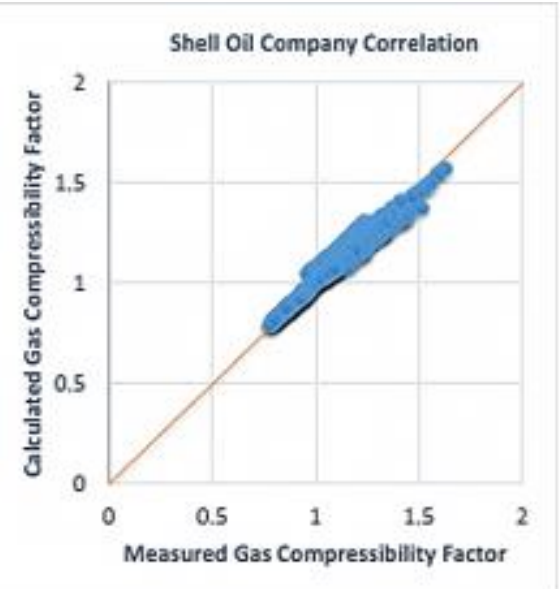

Figure C-2 Accuracy of Shell Oil Company correlation 


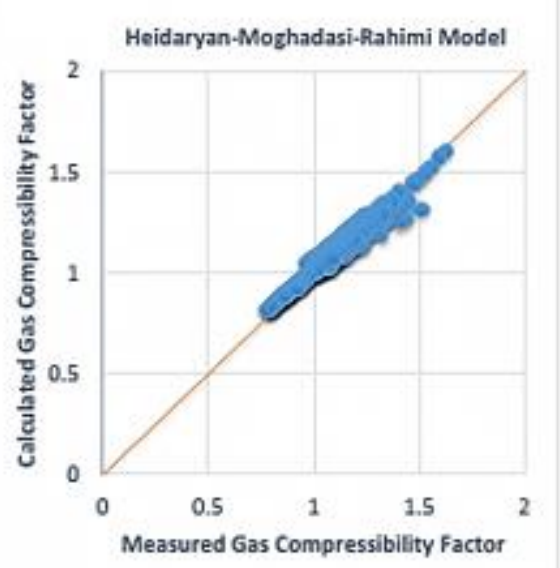

Figure C-3 Accuracy of Heidaryan-Moghadasi-Rahimi model

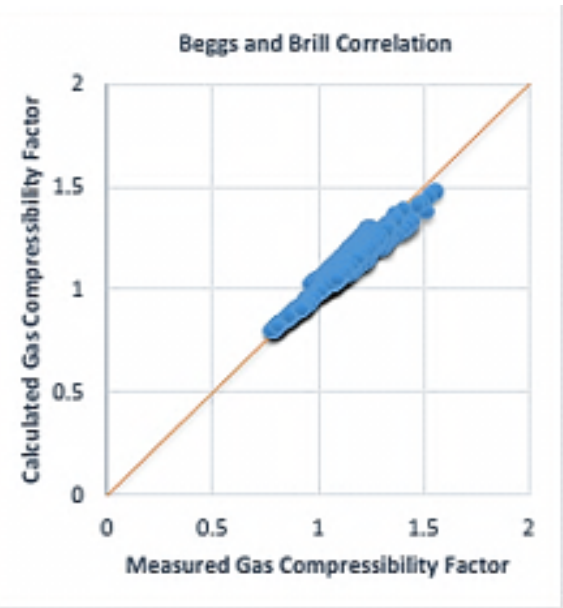

Figure C-4 Accuracy of Brill and Beggs correlation

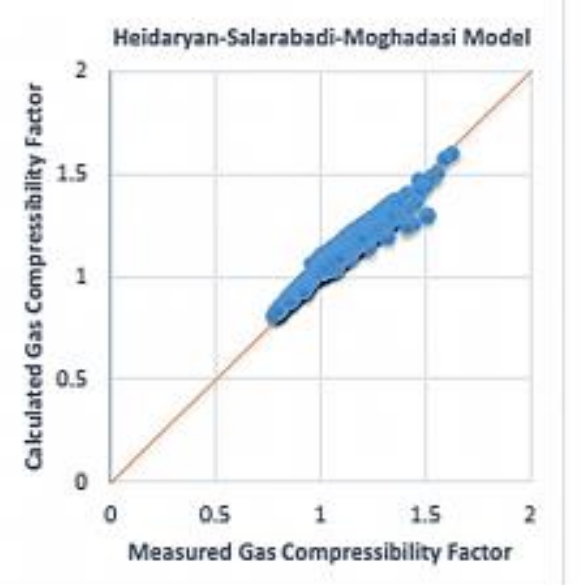

Figure C-5 Accuracy of Heidaryan-Salarabadi-Moghadasi model

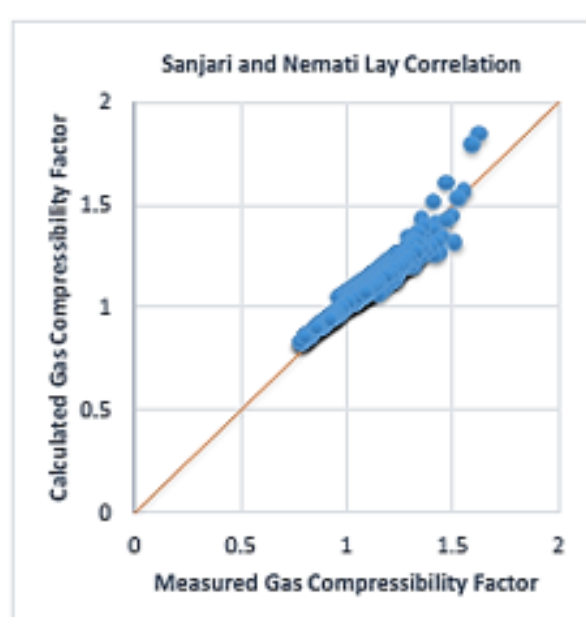

Figure C-6 Accuracy of Sanjari and Nemati Lay correlation 